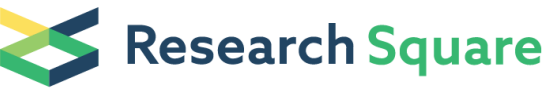 \\ Preprints are preliminary reports that have not undergone peer review. \\ They should not be considered conclusive, used to inform clinical practice, \\ or referenced by the media as validated information.
}

\section{Compositional analysis of the bacterial community in colostrum samples from women with gestational diabetes mellitus and obesity}

\section{July Stephany Gámez-Valdez}

Instituto Tecnologico y de Estudios Superiores de Monterrey Escuela de Ingeniería y Ciencias

José Francisco García-Mazcorro

MNA de Mexico, Research and Development

Alan Heriberto Montoya Rincón

Instituto Tecnológico y de Estudios Superiores de Monterrey, Escuela de Medicina y Ciencias de la Salud

Dalia Liliana Rodríguez Reyes

Instituto Tecnológico y de Estudios Superiores de Monterrey, Escuela de Medicina y Ciencias de la Salud

Gelacio Jiménez Blanco

Instituto Tecnológico y de Estudios Superiores de Monterrey, Escuela de Medicina y Ciencias de la Salud

Melissa Tatiana Alanís Rodriguez

Instituto Tecnologico y de Estudios Superiores de Monterrey Escuela de Medicina y Ciencias de la Salud

Rebeca Pérez-Cabeza de Vaca

Centro Medico Nacional 20 de Noviembre

Mario René Alcorta García

Instituto Tecnológico y de Estudios Superiores de Monterrey, Escuela de Medicina y Ciencias de la Salud

Marion Emilie Genevieve Brunck

Instituto Tecnológico y de Estudios Superiores de Monterrey, Escuela de Ingeniería y Ciencias

Víctor Javier Lara Díaz

Instituto Tecnológico y de Estudios Superiores de Monterrey, Escuela de Medicina y Ciencias de la Salud cuauhtemoc licona-cassani ( $\square$ clicona@tec.mx )

Instituto Tecnologico y de Estudios Superiores de Monterrey - Campus Eugenio Garza Sada https://orcid.org/0000-0002-0360-3945

Research article

Keywords: Breastmilk Microbiota, Gestational Diabetes Mellitus, Obesity, Colostrum, 16S sequencing

Posted Date: November 13th, 2020

DOl: https://doi.org/10.21203/rs.3.rs-48912/v2 
License: (c) (i) This work is licensed under a Creative Commons Attribution 4.0 International License. Read Full License 


\section{Abstract}

Background. Gestational Diabetes Mellitus (GDM) and obesity are threatening health conditions during pregnancy, as they affect the normal function of multiple systems, including neuro-hormonal networks, and adipose, liver, muscle and placenta. GDM and maternal obesity are main triggers to a vicious cycle of metabolic and cardiovascular diseases perpetuated trans-generationally. One of the first stages of this vicious cycle occurs during early lactation as the infant feeds of "disbalanced" breastmilk microbiota and macromolecule level. Despite the importance of breastmilk microbiota on newborn development, few studies have characterized breastmilk microbiota in association with obesity and GDM. Maternal obesity decreases the diversity of breastmilk microbiota, with increased proportions Staphylococcus compared to Bifidobacterium and Bacteroides. However, the extent to which GDM together with maternal obesity affect breastmilk microbiota is unknown. Here, we applied 16S-rRNA high-throughput sequencing to characterize colostrum microbiota of 43 mothers with exclusive GDM and obesity in order to address the impact of GDM/obesity on breastmilk microbiota.

Results. We identified a total of 1,496 amplicon sequence variants (ASVs), being Proteobacteria and Firmicutes the dominant phyla. We found Staphylococcus, Corynebacterium-1, Anaerococcus and Prevotella overrepresented in samples of women with obesity and women with GDM. Population diversity indicators, such as Shannon/Faith phylogenetic index and UniFrac/robust Aitchison distances show distinct microbial composition for GDM (female-newborn subgroup) and obesity (male-newborn subgroup) compared to controls. Finally, Differential abundance analysis showed that Rhodobacteraceae was distinct for GDM and five families (Bdellovibrionaceae, Halomonadaceae, Shewanallaceae, Saccharimonadales and Vibrionaceae) were distinct for obesity groups.

Conclusions. Our study indicates that GDM and obesity are related to a higher microbial diversity. Preliminary functional analysis suggests that this could be attributed to overrepresentation of amino acid and carbohydrate metabolism bacterial pathways. However, there is a strong influence of the infant's gender and the utilization of antibiotics intrapartum. To our knowledge, this study represents the first characterization of the taxonomical changes of colostrum breastmilk in mothers with GDM. We think this study contributes to future design of functional metagenomic studies aiming to understand molecular mechanisms by which breastmilk from mothers with GDM/obesity affect the development and future adult physiology of their suckling infants.

\section{Background}

Breastfeeding during the first semester of life is crucial as the newborn's gastrointestinal tract matures and develops determinants of future health $[1,2]$. In addition to nutritional components (proteins, oligosaccharides, short-chain fatty acids) [3], breastmilk contains a diverse microbial population which participates in educating the gastrointestinal immune system and affects the infant gut-brain axis [4-6]. The so-called breastmilk microbiota contains up to $10^{6}$ bacterial cells / $\mathrm{mL}$ and almost 2,000 of Amplicon Sequence Variants (ASVs) under healthy conditions $[7,8]$. Relative abundance and taxonomical 
distribution of the breastmilk microbial population is shaped by ethnicity, diet [9], body mass index (BMI) [10], inflammation of breast tissue (mastitis) [11], antibiotic use [12] and health condition during the gestational period $[13,14]$.

Gestational diabetes mellitus (GDM) is a pregnancy complication with $17 \%$ incidence worldwide where women develop chronic hyperglycemia during the gestational period [15]. Risk factors include advanced maternal age, diet micronutrient deficiency, and overweight/obesity [16]. In addition, short-term impact of GDM includes an increased risk of type 2 diabetes and cardiovascular disease in both the mother and the infant during adulthood. While molecular mechanisms are still missing, GDM and maternal obesity are strongly correlated to higher incidence of allergic manifestations and metabolic-related diseases including atopic dermatitis (7.5-fold increase) allergen sensitization (5.9-fold increase) $[17,18]$. Most importantly, GDM is considered the bottom-line of a trans-generational vicious cycle of obesity and diabetes affecting the entire population and have a huge economic impact on the public health system worldwide.

The molecular processes underlying the pathophysiology of GDM encompass multiple systems and organs including neurohormonal networks, adipose tissue, liver, muscle and placenta. One of the most known effects of GDM is b-cell dysfunction during pro-insulin synthesis, post-translational modifications, granule storage or sensing peripheral glucose concentrations [16]. Another important molecular mechanism during GDM is the increased number of resident adipose tissue macrophages secreting proinflammatory cytokines such as TNF-a, IL- 6 and IL-1b which are related to impair the release of insulin from b-cells [16] Finally, it has been shown that placenta of mothers with GDM contains lower eosinophil leukocytes count and lower placental expression of the immune mediators IL-10 and TIMP3 compared to normoglycemic controls [19].

Despite the importance of a well-balanced breastmilk microbiota on newborn development, limited studies have focused on characterizing breastmilk bacterial profiling and its microenvironment in association with obesity and GDM. For instance, pre-conceptional obesity $\left(B M I>30 \mathrm{~kg} / \mathrm{m}^{2}\right)$ is linked to a less diverse breastmilk microbial population with increased proportions of Staphylococcus compared to the Bifidobacterium and Bacteroides [20-22]. At the nutritional level, several studies show significant changes in breastmilk of diabetic pregnancies through all the lactating phases. For example, altered levels of anti-infective proteins such as lactoferrin, glycosylation of slgA suggesting that glucose dysregulation has consequences that affect macromolecular composition of breastmilk [23]. Unfortunately, there is no data available about microbiome changes of breastmilk from diabetic pregnancies.

Here, we report a compositional analysis of the microbiota of colostrum samples of Mexican individuals with GDM and obesity. We used a cohort study including mothers with obesity (non-GDM) and mothers with GDM with a biomass index lower than $30 \mathrm{~kg} / \mathrm{m}^{2}$ (non-obese) from Monterrey, the Mexican city with the highest affection of GDM [24]. We sampled colostrum within the first $24 \mathrm{~h}$ after birth and used a $16 \mathrm{~S}$ rRNA amplicon sequencing approach to identify changes at the taxonomical level. The work presented is 
pioneering on the characterization of the microbial population in colostrum of women with obesity and GDM. Most importantly, our results represent the first step towards a molecular mechanistic explanation of interactions between the breastmilk microbial population under GDM and obesity.

\section{Results}

Breastmilk samples (colostrum) from 43 Mexican individuals aged 20-32 years were used in this study. All deliveries were at term with a mean of 39.4 weeks of gestation. A total of 18 samples from mothers with $\mathrm{BMI}<25 \mathrm{~kg} / \mathrm{m}^{2}$ (non-obese), with no GDM symptoms were considered as controls. Twelve milk samples were collected from mothers with obesity $\left(B M I \geq 30 \mathrm{~kg} / \mathrm{m}^{2}\right)$ and without GDM, as well as 13 samples were obtained from mothers with GDM and a BMI $<30 \mathrm{~kg} / \mathrm{m}^{2}$. None of the participants in the gestational diabetes study group had obesity; however, eight of them were overweight (BMI $\left.\geq 25 \mathrm{~kg} / \mathrm{m}^{2}\right)$. Most of our participants were multiparous and 25 women were given antibiotics during labor. Regarding delivery, 24 neonates were born by caesarean section and the sex distribution of the newborns was $48.8 \%$ of males and $51.16 \%$ of females. Clinical and demographics of the participants are summarized in Table 1.

Table 1. Clinical characteristics of subjects included in the study $(n=43)$. 


\begin{tabular}{|c|c|c|}
\hline & $\mathbf{n}$ & Values \\
\hline Maternal age (years) & 43 & $24.6 \pm 3.4$ \\
\hline \multicolumn{3}{|l|}{ Maternal BMI $\left(\mathrm{Kg} / \mathrm{m}^{2}\right)$} \\
\hline Non-obese (20-29.9) & 31 & $23.8 \pm 2.6$ \\
\hline Obese $(\geq 30)$ & 12 & $33.2 \pm 3.0$ \\
\hline \multicolumn{3}{|l|}{ Gestational age (weeks) } \\
\hline Term & 43 & $39.4 \pm 1.3$ \\
\hline Preterm & 0 & $0.0 \pm 0.0$ \\
\hline \multicolumn{3}{|c|}{ Problems during pregnancy } \\
\hline None & 22 & $51.2 \%$ \\
\hline Gestational diabetes & 13 & $30.2 \%$ \\
\hline Urinary tract infection & 9 & $20.9 \%$ \\
\hline Cervicovaginitis & 6 & $14.0 \%$ \\
\hline \multicolumn{3}{|l|}{ Mode of delivery } \\
\hline Vaginal & 19 & $44.2 \%$ \\
\hline Cesarea & 24 & $55.8 \%$ \\
\hline \multicolumn{3}{|l|}{ Parity } \\
\hline 1 & 10 & $23.3 \%$ \\
\hline$\geq 2$ & 33 & $76.7 \%$ \\
\hline \multicolumn{3}{|c|}{ Maternal exposure to antibiotics } \\
\hline During delivery & 25 & $58.1 \%$ \\
\hline None & 18 & $41.9 \%$ \\
\hline \multicolumn{3}{|l|}{ Sex of the baby } \\
\hline Female & 22 & $51.2 \%$ \\
\hline Male & 21 & $48.8 \%$ \\
\hline
\end{tabular}

We classified data according to the pathophysiological condition and the newborn gender resulting in a total of six study subgroups, namely: obesity positive, GDM negative - female newborn (Ob-F; $n=8)$; obesity positive, GDM negative - male newborn (Ob-M; $n=4)$; GDM positive, obesity negative - female newborn (GD-F; n=6); GDM positive, obesity negative - male newborn (GD-M; $n=7$ ); healthy normal weight - female newborn (NW-F; $n=8)$ and healthy normal weight - male newborn (NW-M; $n=10)$.

After birth, breastmilk was collected within the first 24 hours and colostrum was appropriately stored at $-20^{\circ} \mathrm{C}$ until analysis. Using NGS, $1,675,157$ high quality reads were obtained with a mean of $38,957 \pm$ 27,723 sequencing reads per sample. Removal of possible contaminants and rare taxa was performed through an exhaustive batch analysis for all samples processed. We identified batch effect for 
Pseudomonas, Enterobacteriaceae, Ralstonia and Herbaspirillum across our dataset. Even though Pseudomonas and Enterobacteriaceae are part of other breastmilk microbial compositional studies [8,25-28] we decided to remove them from our dataset as clear batch effect is observed (Supplementary Figure 1).

After the removal of possible contaminants and rare taxa ( $\leq 25$ reads in total), 1,496 amplicon sequence variants (ASVs) were assigned at 30 phyla, 58 classes, 133 orders, 217 families, 395 genera and 335 species using Silva 132 database with a confidence identity level set at $99 \%$. We found increased prevalence of Staphylococcus, Corynebacterium 1, Anaerococcus and Prevotella in samples from participants with GDM and obesity. Detailed information about the abundance of ASVs found per sample can be found in the Supplementary Table 1.

\section{Colostrum compositional microbiota was dominated by Staphylococcus in all the samples.}

We found that breastmilk samples were overrepresented by the family member of the Firmicutes phylum Staphylococcaceae with a relative abundance mean of $13.9 \% \pm 16.5 \%$ (range $0.1 \%-76.3 \%$ ). Followed by the Proteobacteria phylum members Rhizobiaceae with a relative abundance mean of $10.3 \% \pm 11.1 \%$ (range $0.1 \%-47.2 \%$ ) and Burkholderiaceae $(9 \% \pm 11.7 \%$ (range $0 \%-48.6 \%$ ). Less represented phyla were Bacteroidetes with Weeksellaceae $(8.6 \% \pm 11.9 \%$ (range $0 \%-37.2 \%)$ and Prevotellaceae $(3.8 \% \pm 7.1 \%$ (range $0 \%-24.3 \%$ ) and Actinobacteria with Corynebacteriaceae $(3.2 \% \pm 4.5 \%$ (range $0 \%-16.2 \%)$. The classification of "Other" represents phyla with less than $1 \%$ of total relative abundance (Figure 1A).

Bacteria belonging to the Burkholderiaceae family were more abundant in obesity-male (Ob-M, $21.9 \% \pm$ $9.5 \%$ ) but less abundant in obesity-female (Ob-F, $2 \% \pm 4.5 \%)$. The highest relative abundance of

Staphylococcaceae, Streptococcaceae and Bacillales was shown for obesity-female (Ob-F, $24.2 \% \pm 25.8 \%$; $13.9 \% \pm 19.3 \% ; 12.6 \% \pm 13.6)$. Xanthobacteraceae showed higher abundance in all the female groups compared to their male counterpart. Sphingomonadaceae were more abundant in healthy groups with $8.4 \% \pm 6.5 \%$ for female (NW-F) and $10 \% \pm 7.2 \%$ for male (NW-M). This behavior was also observed for WPS-2, a recent candidate division, which had the highest relative abundance in healthy-female (NW-F, $5.5 \% \pm 10 \%$ ) (Figure $1 \mathrm{~A})$.

\section{Staphylococcus, Corynebacterium 1, Anaerococcus and Prevotella are overrepresented in samples of individuals with obesity and GDM}

Overall, the most abundant genera were Staphylococcus $(13.9 \% \pm 16.5 \%)$, Chryseobacterium $(7.9 \% \pm$ $11.6 \%)$, Rhizobiaceae (7.3\% $\pm 9.2 \%)$, Streptococcus (7.1\% $\pm 16.1 \%)$, Sphingomonas $(6.6 \% \pm 6 \%)$ and Burkholderia (5.5\% $\pm 9.8 \%$ ) (Figure 2). The "Other" category represents the taxa with less than $1 \%$ overall. According to the subgroup classification, in the healthy normal weight subgroups, Chryseobacterium $(\mathrm{p}<$ $0.05)$ and Sphingomonas $(\mathrm{p}<0.10)$ were more abundant $(15 \% \pm 16.5 \%, 8 \% \pm 6.5 \%$ for female (NW-F) and $12 \% \pm 13.7 \%, 9.3 \% \pm 6.7 \%$ for male (NW-M) respectively). Corynebacterium $1(\mathrm{p}<0.10)$ and Anaerococcus $(\mathrm{p}<0.10)$ were more abundant in subgroups with obesity and GDM. Staphylococcus was more abundant $(p<0.05)$ in obese and GDM subgroups with female baby $(O b-F, 24.2 \% \pm 25.8 \%$; GD-F, 
$15.5 \% \pm 11.9 \%)$ compared to their healthy counterpart. Obesity-female $(\mathrm{Ob}-\mathrm{F})$ had the highest prevalence of Gemella $(\mathrm{p}<0.05)$. While not statistically significant, Streptococcus and Neisseria presented higher relative abundance in obesity-female (Ob-F) compared to the rest of subgroups. Stenotrophomonas and Burkholderia were more abundant in subgroups with male neonate compared to the healthy counterpart ( $p<0.05)$, distinctively in obese $(\mathrm{Ob}-\mathrm{M}, 6.6 \% \pm 5.7 \%$ and $16.7 \% \pm 7.1 \%$ respectively). A similar pattern was also observed for Prevotella in the obesity-male $(p<0.05)$ and in the GDM-male $(p<0.10)$ subgroups. Breastmilk from subjects with GDM showed higher prevalence of "Other genera" (29.8\% $\pm 13 \%, 25.9 \% \pm$ $7.4 \%$ for female and male respectively), suggesting that the biggest part of this contribution to the relative abundance of both subgroups is due to genera with less than $1 \%$ in total. This is supported by the rarefaction curves which reveal that GDM-female (GD-F), GDM-male (GD-M) and obesity-female (Ob-F) had higher values of estimated number of observed ASVs (Figure 1B). A full distribution of taxa is shown in Figure 2.

\section{Alpha and beta diversity metrics show a distinct microbial composition for GD-F, Ob-M and newborn gender-related samples}

We used a general linear model $(\mathrm{glm})$ using alpha diversity metrics at a sequencing depth of 6,130 (data not shown) in order to quantify the influence of GDM, obesity $\left(B M I \geq 30 \mathrm{~kg} / \mathrm{m}^{2}\right)$, mode of delivery, antibiotic exposure, multiparity and sex of the baby. As a result of the analysis, maternal physiopathology (GDM, obesity and healthy) and antibiotic exposure showed statistically significant association ( $\mathrm{p} \leq$ $0.10)$ for Shannon index and observed ASVs. The implementation of intrapartum antibiotics was related to a decrease in diversity. The sex of the baby showed statistically significant association $(p \leq 0.05)$ for phylogenetic diversity and observed ASVs (Figure 3A-C). GDM subgroups presented the highest values in all alpha indexes. In addition, our results suggest that, in general, female subgroups had higher diversity compared to male subgroups. Fisher's comparisons indicate that statistical difference was only significant between healthy-female (NW-F) and GDM-female (GD-F) for Shannon index. Breastmilk samples from obesity-male subgroup (Ob-M) had the lowest levels of alpha diversity and were statistically different to all the subgroups, including their female counterpart (Figure 3A-C).

We estimated microbiome beta diversity using the unweighted UniFrac distance (Figure 3D). Our results show that obesity-male (Ob-M) subgroup cluster separately from the rest of the samples (PERMANOVA; $p$ $=0.047 ; 999$ permutations). Using the unweighted distance matrix, we generated a PCoA biplot in order to show that the clustering was significant for obesity-male (Ob-M; $\mathrm{p}<0.05)$ compared to healthy-male (NW$M)$. Arrows in the plot represent the correlation at family level with PCoA axes, indicating their contribution to the variation (Figure 3D). While samples from GDM-female (GD-F), GDM-male (GD-M), healthy-female (NW-F) and obesity-female (Ob-F) show high similarity regarding microbial composition, the unweighted measurement indicates that there is a phylogenetic difference between obesity-male (Ob$M)$ and the rest of subgroups $(p<0.05)$.

We used the beta-diversity compositional Aitchison's distance in order to assess the compositional nature of data (PERMANOVA; $p=0.002 ; 999$ permutations) (Figure 3E). The robust principal component analysis 
(RPCA) biplot, which allows to examine the variation of samples and taxa, did not show a clear separation of any subgroup. PERMANOVA tests and pairwise comparisons showed that obesity-male $(\mathrm{Ob}-\mathrm{M})$ was different to both GDM subgroups $(\mathrm{p}<0.05)$ and that obesity-female subgroup $(\mathrm{Ob}-\mathrm{F})$ was statistically different to its male counterpart $(\mathrm{Ob}-\mathrm{M})$ and both healthy and GDM subgroups $(p<0.05)$. In addition, the GDM-male (GD-M) subgroup was different to its healthy counterpart (NW-M; $p<0.10)$. The 7 taxa presented as vectors in the plot are the most significant drivers of the location of samples (Figure $3 \mathrm{E})$.

\section{Breastmilk core and differentially abundant taxa}

We defined the breastmilk core microbiota as taxonomical families present in all samples with a minimum $1 \%$ of total mean relative abundance. Overall, 8 families were identified as the core taxa and comprise $56.8 \% \pm 11.3 \%$ of the total (Table 2). The most abundant were Staphylococcaceae with a general mean of $13.9 \% \pm 16.5 \%$, followed by Rhizobiaceae $(10.3 \% \pm 11.1 \%)$, Burkholderiaceae $(9 \% \pm$ $11.7 \%)$ and Streptococcaceae $(7.1 \% \pm 16.1 \%)$. These results demonstrate the high variability of the core bacteria among subgroups and individuals. The four most abundant families belonging to the core were found to describe most of the variation in the ordination space observed in the unweighted PCoA biplot and were represented as arrows (Figure 3D). However, no clear participation of families to the formation of subgroups was visualized with the implement of UniFrac metrics.

Table 2. Breastmilk core microbiota at the taxonomical family level (\% relative abundance \pm standard deviation).

\begin{tabular}{|c|c|c|c|c|c|c|c|}
\hline Core family & Overall (\%) & $\begin{array}{l}\text { Healthy-Female } \\
\text { (NW-F) }\end{array}$ & $\begin{array}{c}\text { Healthy-Male } \\
\text { (NW-M) }\end{array}$ & $\begin{array}{c}\text { Obesity-Female } \\
\text { (Ob-F) }\end{array}$ & $\begin{array}{c}\text { Obesity-Male } \\
\text { (Ob-M) }\end{array}$ & $\begin{array}{c}\text { GDM-Female } \\
\text { (GD-F) }\end{array}$ & $\begin{array}{c}\text { GDM-Male } \\
\text { (GD-M) }\end{array}$ \\
\hline Staphylococcaceae & $13.9 \pm 16.5$ & $9.9 \pm 14.9$ & $4.7 \pm 4.6$ & $24.2 \pm 25.8$ & $7.6 \pm 7.0$ & $15.6 \pm 11.9$ & $21.9 \pm 16.9$ \\
\hline Rhizobiaceae & $10.3 \pm 11.1$ & $7.7 \pm 3.7$ & $9.8 \pm 12.6$ & $9.1 \pm 11.7$ & $5.5 \pm 2.8$ & $22.9 \pm 16.6$ & $7.1 \pm 4.5$ \\
\hline Burkholderiaceae & $9.0 \pm 11.7$ & $8.8 \pm 9.0$ & $11.5 \pm 14.7$ & $2.0 \pm 4.5$ & $21.9 \pm 9.5$ & $7.2 \pm 15.4$ & $7.8 \pm 9.3$ \\
\hline Streptococcaceae & $7.1 \pm 16.1$ & $6.6 \pm 17.1$ & $12.5 \pm 23.4$ & $13.9 \pm 19.3$ & $1.3 \pm 1.9$ & $1.8 \pm 1.5$ & $0.4 \pm 0.2$ \\
\hline Sphingomonadaceae & $7.1 \pm 6.3$ & $8.4 \pm 6.5$ & $10.0 \pm 7.2$ & $4.6 \pm 6.2$ & $6.4 \pm 3.9$ & $4.3 \pm 2.2$ & $6.9 \pm 8.0$ \\
\hline Xanthomonadaceae & $4.6 \pm 6.1$ & $5.1 \pm 4.4$ & $6.8 \pm 8.1$ & $1.5 \pm 2.4$ & $7.1 \pm 5.5$ & $1.1 \pm 1.1$ & $6.1 \pm 8.6$ \\
\hline Prevotellaceae & $3.8 \pm 7.1$ & $4.8 \pm 8.2$ & $2.2 \pm 4.5$ & $0.4 \pm 0.4$ & $6.1 \pm 10.9$ & $4.9 \pm 8.0$ & $6.8 \pm 10.0$ \\
\hline Bacillaceae & $1.0 \pm 1.5$ & $1.4 \pm 3.0$ & $1.3 \pm 1.7$ & $0.8 \pm 0.7$ & $0.4 \pm 0.2$ & $1.0 \pm 0.6$ & $0.9 \pm 0.5$ \\
\hline $\begin{array}{c}\text { Total relative } \\
\text { abundance (\%) }\end{array}$ & $56.8 \pm 11.3$ & $52.7 \pm 9.5$ & $58.8 \pm 11.8$ & $56.4 \pm 14.2$ & $56.3 \pm 8.3$ & $58.8 \pm 11.5$ & $58.0 \pm 10.4$ \\
\hline
\end{tabular}

We used the Aldex2 tool [29] in order to identify differences in ASV abundance between subgroups. We determined the taxa that were driving the difference between the subgroups and obtained effect plots (based on the effect size), which allowed us to visualize if the variation was higher between or within subgroups. Given the high variability amongst samples, we only observed differentially abundant ASVs with a significant expected Benjamini-Hochberg corrected $p$-value of Welch's $t$ test $(q \leq 0.1)$ in three sample pairs (Figure 4). In the GDM-female vs healthy-female (GD-F vs NW-F) comparison, the family 
Rhodobacteraceae was identified as different ( $q<0.10$; Figure 4A). While in the healthy-male vs obesitymale (NW-M vs Ob-M) were found a total of 5 families (Vibrionaceae, Halomonadaceae, Shewanallaceae, Bdellovibrionaceae, and Saccharimonadales) with an absolute effect size greater than 1 and a q-value $\leq$ 0.05 (Figure 4B), in the obesity-male vs obesity-female (Ob-M vs Ob-F) 10 significant taxa were different, of which only 2 (Burkholderiaceae and Sphingobacteriaceae) corresponded to the core families (Figure 4C). Based on the median difference between subgroups, we observed that in all comparisons, obesitymale (Ob-M) had significant higher abundance for differential taxa found. In addition, GDM-female (GDF) had higher prevalence of Rhodobacteraceae compared to healthy-female (NW-F).

\section{Discussion}

We present the first compositional study of colostrum microbiota of individuals with obesity (BMI $\geq 30$ $\mathrm{kg} / \mathrm{m}^{2}$ ) and gestational diabetes mellitus. Colostrum is supposedly the first postnatal maternal fluid in contact with newborns' gastrointestinal system; therefore, identifying differences in its microbial composition could directly explain aberrant function of the infant's immune gastrointestinal system. Overall, our results show that colostrum microbial composition are overrepresented with Staphylococcaceae and Rhizobiaceae taxonomic families (Figure 1A). Staphylococcus, Chryseobacterium, Streptococcus and Sphingomonas were the most abundant genera in our data set, which correlates with the microbial composition of breastmilk from women from Mexico, Taiwan, Finland and China (Beijing area) $[25,28,30]$. Other datasets showed Pseudomonas and Staphylococcus as predominant taxa in breastmilk samples from Spanish and Irish individuals in addition to commensal and obligate anaerobes such as Bifidobacterium and Bacteroides [27,31].

In general, low-biomass samples are unstable and their relative abundance depends on subtle changes of sample handling or sequencing methodologies [32,33]. More importantly, low-biomass samples are prone to DNA extraction and library preparation kit contaminants [34]. Different studies show that presence of Pseudomonas and Ralstonia in breastmilk could be the result of reagent contamination effect, especially when culturomics approaches fail to isolate such microorganisms in selective culture media [31]. Alternative to the utilization of negative controls, batch processing for all the samples allows for visual inspection of contaminants. In our study, samples were processed for DNA purification in a total of 16 batches. Detailed analysis allowed us to identify batch effect across our dataset for Pseudomonas, Enterobacteriaceae, Ralstonia and Herbaspirillum (Supplementary Figure 1). Even though such taxonomic groups have been reported in different breastmilk studies, including in a cohort of Mexican individuals $[8,25,27,28]$, we decided to remove them from our dataset.

The candidate division WPS- 2 was present in $2.1 \%$ of total relative abundance in our samples. This phylum has been described in human and canine oral microbiota and soil [35-39]. WPS-2 was incorporated in the Human Oral Microbiome database (HOMD) in 2014 [40]. While it has not yet been described in microbiota of newborns' oral cavity, WPS-2 was present in nasopharynx samples from infants under 6 months of age [41]. This is the first time that WPS- 2 is reported in breastmilk samples 
with high prevalence supporting the retrograde flux theory, in which microbiota from neonate's mouth contributes to the settlement of breastmilk bacteria [42].

In addition, we identified that breastmilk microbial diversity was specific to the newborn's sex $(p \leq 0.05)$. Our results are in accordance with previous reports where BMI and neonate's gender were related to enrichment of Staphylococcus, Stenotrophomonas and Burkholderia [8]. Regardless of the pathology (GDM or BMI), female-related colostrum samples showed higher alpha diversity compared to male subgroups, suggesting a more diverse microenvironment (Figure 3A-C). It has been demonstrated that gut and oral microbiota from children and maternal breastmilk biochemical composition differ between female and male infants, possibly due to variation in hormone recruitment and energetic demand during pregnancy $[8,43-45]$. According to this, we strongly suggest that neonate microbiota sex-bias should be an important consideration for further experimental designs trying to explain causality microbial changes due to any pathology.

Despite the sample heterogeneity, we guided our analysis by a general linear model to show that colostrum samples of individuals with obesity (Ob-F and Ob-M) or GDM (GD-F and GD-M) are enriched for Staphylococcus ( $\mathrm{p} \leq 0.10$ ) compared to their healthy normal weight counterpart (Figure 2 ). It has been observed that higher numbers of Lactobacillus and Staphylococcus were related to higher maternal BMI $[21,46,47]$. In addition, decreased Streptococcus abundance and an increment in breastmilk microbial diversity in Mexican-American subjects with high BMI $\left(>25 \mathrm{~kg} / \mathrm{m}^{2}\right)$ has been previously reported [10]. These results also correlate with our results, where Streptococcus are less abundant and high values of observed ASVs were found in obesity-male (Ob-M, 167ASVs) and obesity-female (Ob-F, 295 ASVs) subgroups. However, other reports show that colostrum samples from obese mothers presented a less diverse microbiota compared to non-obese samples [21]. This variability can be attributed to differences in study populations (geographical location, diet, socioeconomic status), sample collection at different lactation stage and neonate sex-bias.

We implemented the Aldex 2 tool, which performs a log transformation and replacement of the zero values in the obtained results for a matrix creation that allows the determination of significant differences of taxa between subgroups [29]. Interestingly, we observed Bdellovibrionaceae and Saccharimonadales, which are ultra-small parasite bacteria, differentially present in Ob-M compared to its corresponding healthy (NW-M) contrast (Figure 4B). While further research is needed, this pattern can be attributed to resilience mechanisms of breastmilk microenvironment to maintain a functional equilibrium through specific predatory interactions with Gram-negative bacteria such as Burkholderia and Chryseobacterium, which also appear to be in higher proportions in obesity-male (Ob-M; Figure 2). This may be explained by the detection of DNA fragments resulting from the bacterial lysis.

Bdellovibrionaceae has been found in soil, freshwater and human gut from healthy subjects and patients suffering from inflammatory diseases [48]. This taxon is considered as a potential probiotic, since it could modulate the gut biodiversity by its predation of bacteria correlated in chronic inflammatory diseases, such as obesity and Crohn's disease $[49,50]$. On the other hand, Saccharimonadales has been reported in human oral cavity, intestines, skin, and female genital tract [51-53]. Lif et al. [54] related the 
impact of birthing method and a higher prevalence of this novel phylum in oral biofilm samples of infants delivered vaginally compared to infants born by cesarean section. Even though Saccharimonadales remains difficult for cultivation, its presence in adult subgingival plaque, vagina and colon has been associated to human inflammatory mucosal diseases $[55,56]$.

Our results indicate a differential prevalence of Burkholderiaceae and Sphingobacteriaceae in breastmilk samples from obesity-male (Ob-M) compared to its female contrast (Ob-F; Figure 4C). Similar results have been described in oral samples from male infants as they reported a higher abundance of Brachymonas and Sphingomonas [45]. We hypothesize that differences in breastmilk microbiota by infant gender influence the conditioning of the neonate's gut microenvironment for bacterial communities related to the metabolism of nutrients involved in sex-related neurodevelopment.

We observed a higher relative abundance of Staphylococcus, Anaerococcus and Prevotella in both GDM subgroups compared to their corresponding contrast control (NW). Interestingly, similar profiles have been reported for gut microbiota individuals with GDM [57-59]. Complementarily, high prevalence of Prevotella has been observed in oral cavity, amniotic fluid and gut microbiota of pregnant women with GDM, which confirms vertical transmission mother-to-baby and supports the enteromammary theory of breastmilk microbiota origin [57,60-62]. Functional metagenomic approaches are needed in order to determine what is the exact role of Prevotella as keystone taxa under the specific microenvironment shaped by GDM. Rhodobacteraceae was observed in higher proportion in GDM-female (GD-F) compared to healthy-female (NW-F) (adjusted p-value < 0.1). Rhodobacteraceae has been mostly reported in soil [63], but also in breastmilk from healthy Mexican women [25], human skin [64], meconium [65] and fecal samples from patients suffering from diarrhea [66].

In order to attempt to provide a prelaminar functional insight for the taxonomic profiles observed in our study, we used the Phylogenetic Investigation of Communities by Reconstruction of Unobserved States (PICRUSt2) software [67] and Linear Discriminant Analysis (LDA) Effect Size (LEfSe) [68]. A total of 24 metabolic pathways reported in MetaCyc database [69] were considered as significant ( $p$-value $<0.05$, LDA score > 3.5). While the results suggested that bacterial pathways involved in amino acid and carbohydrate metabolism were significantly represented in the obese and with GDM subgroups (Figure $5)$.

Despite that higher levels of branched-chain amino acids (BCAA) valine, leucine and isoleucine have been described in blood from GDM woman and have been linked to the early pathogenesis of type 2 diabetes [70-72], lactation for more than 3 months in women with GDM has been associated with changes in the BCAA profile. However, the mechanism that explains this protection role is still unclear $[71,73]$. Palmitate biosynthesis and inositol isomers degradation pathways were more prevalent in GDM-female (GD-F) subgroup. Palmitate contains palmitic acid which is one of the major saturated fatty acids in colostrum, it provides around $25 \%$ of milk fatty acids and it is involved in absorption of fat and calcium [74]. Accordingly, GDM and obesity have been related to higher levels of this free fatty acid in placenta, umbilical cord and breastmilk [75-77]. Myoinositol has an important role in several biological processes 
related to cell survival, lipid metabolism, glycemic control and restoration of ovulation [78]. Women with GDM or polycystic ovary syndrome are deficient in inositol isomers $[79,80]$. Supplementation with myoinositol during pregnancy improves glucose metabolism, reduces the incidence and severity of GDM and decreases adverse neonatal outcomes [78-81]. We acknowledge that prediction of bacterial functional metabolic pathways based on $16 \mathrm{~S}$ amplicon sequencing are strongly biased towards existing reference genomes and caution must be taken in the interpretation of results.

\section{Conclusions}

The factors determining colonization of an infant's gut microbiota is important and could lead to improve health policies. Amplicon sequencing using NGS technologies are the most reliable methods for large-scale microbial compositional studies [82]; however, in order to obtain relevant functional insights, clinical protocols should include dietary habits of the mother, sex of the infant and a strong control of antibiotic usage. Our study indicates that GDM and obesity are related to a higher microbial diversity and significantly overrepresented by amino acid and carbohydrate metabolism bacterial pathways. However, it was specific to the sex of the newborn and affected by the implementation of intrapartum antibiotics. While Prevotella and Anaerococcus are relevant taxonomic groups within the microbial population of colostrum samples of individuals with GDM, Staphylococcus, Corynebacterium 1 and Gemella are overrepresented in colostrum of obese participants. Further studies of the functional role of such taxa are key in order to understand the dynamics of the establishment of infants' gut microbiota under the influence of obesity and GDM. These studies are needed for the potential design of probiotics and in the search of possible therapeutic agents contributing to the infant's gastrointestinal immune system homeostasis.

\section{Material And Methods}

\section{Study population}

Recruiting was done at Hospital Regional Materno Infantil, the main public perinatal medicine hospital of Servicios de Salud del Estado de Nuevo León, Mexico. We included mother-infant pairs, within 20 and 32 years of maternal age, and with a verified address in the Monterrey Metropolitan Area, who accepted the invitation to participate and signed the informed consent document. Exclusion criteria were: mothers who had a history of antibiotic usage in the 3 months prior to delivery; mothers who had prolonged exposure to antibiotics (more than 3 weeks) at any time during pregnancy; mothers who received immunosuppressive or immunomodulatory corticosteroid therapy; history of a vegan, ovolactovegetarian or exclusion diet (e.g.: ketogenic diet); history of bariatric surgery or any complicated surgery; history of feeding disorders; exposure to antineoplastic drugs, histamine-H2 receptor antagonists or proton pump inhibitors and/or monoclonal antibodies; and/or history of diarrhea during the three weeks before delivery. In addition, those with an uncertain last menstrual period date or irregular periods that gave place to an uncertain pregnancy dating were excluded. Elimination criteria were Antibiotics for more than 
24 hours post-delivery; need of intensive care (mother or infant) and/or any condition that impeded recollection of breast milk.

Those selected mother infant pairs were further allocated for analysis to one of the three study groups, according to their $\mathrm{BMI}$ and health condition: 12 obese women (BMI $\left.\geq 30 \mathrm{~kg} / \mathrm{m}^{2}\right), 13$ women suffering from gestational diabetes $\left(\mathrm{BMI} \leq 29.9 \mathrm{~kg} / \mathrm{m}^{2}\right)$ and 18 control healthy women $(\mathrm{BMI} \leq 25 \mathrm{~kg} / \mathrm{m} 2)$. The study groups were further sub-divided by sex of the baby. The study protocol was approved by institutional Review Boards at Escuela de Medicina y Ciencias de la Salud, Tecnológico de Monterrey, with the ID P000185-CarMicrobioLHum2018-CEIC-CR002, on May 6 ${ }^{\text {th }}, 2019$.

\section{Samples collection and processing}

After gentle cleansing only with sterile water of the breasts, each mother performed a gentle circular massage of each breasts until a few drops of colostrum appeared. These first drops were disregarded, and the mother self-expressed approximately $5 \mathrm{~mL}$ (when possible) of colostrum into a sterile falcon-type $20 \mathrm{~mL}$ polypropylene tube, under close medical supervision. The procedure was repeated on the opposite breast. Extreme care was taken to avoid milk contact with breast skin or fingers. Then, the tubes were closed, and kept at $-20^{\circ} \mathrm{C}$ for no more than 48 hours until DNA extraction.

\section{DNA extraction}

Genomic DNA was extracted from $1 \mathrm{~mL}$ of colostrum using an optimized phenol-chloroform protocol [83]. Samples were thawed on ice and centrifugated at $16,000 \mathrm{Xg}$ for 15 minutes, the fat rim was carefully removed, and PBS washes were performed to eliminate fat residues $(0.5 \mathrm{~mL}$ sterile PBS), centrifugation at $16,000 \mathrm{Xg}$ for 10 minutes). The pellet was resuspended in $0.5 \mathrm{~mL}$ of extraction buffer $(220 \mathrm{mM}$ Tris$\mathrm{HCl} \mathrm{pH} \mathrm{7.5,} 110 \mathrm{mM}$ EDTA, $1100 \mathrm{mM} \mathrm{NaCl}, 20 \%$ Triton X-100, 2\% SDS) and $0.3 \mathrm{~mL}$ of $3 \mathrm{M}$ of sodium acetate. An additional step of mechanical lysis was performed by bead-beating with lysing matrix $A$ using a FastPrep (MP Biomedicals, Santa Ana, CA) disruptor at a speed setting of $5.5 \mathrm{~m} / \mathrm{s}$ for $25 \mathrm{~s}$. The lysate was submitted to an enzymatic lysis with $10 \mu \mathrm{L}$ of proteinase $\mathrm{K}(10 \mathrm{mg} / \mathrm{mL}), 5 \mu \mathrm{L}$ of lysozyme $(10$ $\mathrm{mg} / \mathrm{mL}$ ) and $10 \mu \mathrm{L}$ of RNAse, and incubated at $60^{\circ} \mathrm{C}$ for 1 hour. After incubation, $100 \mu \mathrm{L}$ of $1.5 \mathrm{M} \mathrm{NaCl}$ (filter sterile) were added and carefully mixed and maintained at room temperature for 5 minutes. Following incubation, the mixture was centrifugated at $16,000 \mathrm{Xg}$ for 15 minutes and the supernatant was transferred into a new tube and extracted twice with an equal volume of phenol:chloroform:isoamylalcohol (25:24:1). The precipitation of DNA was carried out by the addition of 0.6 volumes of isopropanol and incubation at $-80^{\circ} \mathrm{C}$ for 1 hour. Next, we centrifuged the samples ( $16,000 \mathrm{X} \mathrm{g}$ for 15 minutes), and the isopropanol was retired. The pellet was washed twice with $70 \%$ ethanol, air dried and resuspended in preheated $50 \mu \mathrm{L}$ of nuclease free water. The DNA was measured using a NanoDrop ND-1000 UV spectrophotometer (Thermo Fisher Scientific, Waltham, MA, USA) and the DNA integrity was confirmed through an agarose gel electrophoresis. Unless otherwise specified, all reagents were purchased from Sigma Aldrich. 
In order to identify any contaminations during DNA extractions, we processed all the samples in a total of 16 different batches using freshly prepared solutions prior to run the experiments. Microbial compositional information for all the batches is provided as Supplementary Figure 1.

\section{DNA sequencing and analysis}

DNA samples were sequenced at the Advanced Genomics Unit (LANGEBIO, CINVESTAV) using Illumina MiSeq $(2 \times 300)$ following the $16 \mathrm{~S}$ rRNA amplicon sequencing library preparation (as per manufacturer recommendations) for the amplification of $\mathrm{V} 3-\mathrm{V} 4$ hypervariable region with the universal primers $341 \mathrm{~F}$ 5'CCTACGGGNGGCWGCAG3' and 785(R) 5'GGACTACHVGGGTATCTAATCC 3'. We normalized the DNA for sequencing at $25 \mathrm{ng} /$ هl.

Bioinformatic analyzes were carried out using QIIME 2 v.2019.7 [84]. Sequencing readings were quality filtered using the q2-demux plugin with a minimum length at 270 nucleotides followed by denoising with DADA2 [85]. Single-paired filtered readings were used for the taxonomic species profile using amplicon sequence variants (ASVs) with the q2-feature-classifier [86] against the Silva 132 database with a limit of sequence identity set at $99 \%$ [87]. Removal of potential contaminants include ASVs belonging to Cyanobacteria, Phyllobacterium, Chloroflexi, mitochondria / chloroplast and rare taxa (with less than 25 reads across the entire dataset). Resulting ASVs were aligned with mafft v2019.7 [88] and implemented to create a phylogeny with fasttree2 v2019.7 [89]. Rarefaction of sequences to 4,130 per sample was used to perform alpha and beta diversity analyzes. Observed ASVs, Shannon index and Faith's phylogenetic diversity were used as alpha-diversity metrics; UniFrac (weighted and unweighted) and robust Aitchison distances were used for the creation of PCOA and RPCA respectively. We determined bacterial families present in all samples with a minimum relative abundance of $1 \%$ overall as core microbiota. ASVs that were assigned at family level were considered to perform differential abundance analysis with Aldex2 tool v1.14.1 [29]. We implemented the Phylogenetic Investigation of Communities by Reconstruction of Unobserved States 2 (PICRUSt2) v2.3.0-b software [67] with default options (picrust pipeline.py) to determine the potential link between the microbiome environment and the functional metabolism based on metabolic pathways reported in MetaCyc database [69]. Raw data was deposited and is available at the NCBI Sequence Read Archive (SRA) under SRA accession number PRJNA638389.

\section{Data processing}

QIIME2 a-diversity outputs and ASVs count tables at the family and genus taxonomical level were imported and processed in Minitab 17. Association of observed ASVs, Shannon index, Faith's phylogenetic distance and transformed ASVs count tables with maternal health condition, mode of delivery, antibiotic exposure, parity and gender of the neonate was assessed by general linear model $(\mathrm{glm})$ with $\mathrm{p}$-value of $\leq 0.10$. $\beta$-diversity significance for UniFrac and robust Aitchison distances was calculated using permutational ANOVA (PERMANOVA) with 999 permutations. Differential bacteria were assessed with Welch's $t$ test with a Benjamini-Hochberg's false discovery rate (FDR) p-value correction after a centered log ratio (clr) transformation with a zero-replacement of taxa counts. Functional metabolism prediction results by PICRUST2 were further analyzed in the MicrobiomeAnalyst web-based 
platform [90] using the LEfSe method [68] which performs a Kruskal-Wallis rank sum test to determine the significantly different features between groups and a linear discriminant analysis (LDA) to estimate their effect size. A metabolic pathway was considered significant with a p-value $\leq 0.05$ and an LDA score $\geq 3.5$.

\section{List Of Abbreviations}

GDM: Gestational diabetes mellitus

ASV: Amplicon sequence variant

BMI: Body mass index

FDR: False discovery rate

IL-10: Interleukin 10

TIMP3: Tissue inhibitor of metalloproteinase 3

WHO: World Health Organization

OECD: Organisation for Economic Cooperation and Development

NW-F: Healthy normal weight - female neonate

NW-M: Healthy normal weight - male neonate

Ob-F: Obesity positive GDM negative - female neonate

Ob-M: Obesity positive GDM negative - male neonate

GD-F: Gestational diabetes mellitus positive obesity negative - female neonate

GD-M: Gestational diabetes mellitus positive obesity negative - male neonate

FDR: False discovery rate

BCAA: Branched-chain amino acids

GLM: General linear model

PCoA: Principal coordinate analysis

RPCA: Robust principal component analysis

PERMANOVA: Permutational ANOVA 
PBS: Phosphate buffered saline

EDTA: Ethylenediaminetetraacetic acid

SDS: Sodium dodecyl sulfate

\section{Declarations}

\section{Ethics approval and consent to participate}

The clinical protocol was approved by the Ethics Committee of the Escuela de Medicina y Ciencias de la Salud (TecSalud) (Project ID: P000185-CarMicrobioLHum2018-CEIC-CR002).

\section{Consent for publication}

All authors have read the manuscript and have provided their consent for publication.

\section{Availability of data and materials}

Raw data is available in the NCBI under ID number 638389 and Bioproject accession number PRJNA638389.

\section{Competing interests}

All authors have read the manuscript and declare no conflict of interest.

\section{Funding}

The project was funded under ITESM-0021C21064 seed grant.

\section{Authors' contributions}

PCVR, BM, LDVJ and LCC designed all experiments and clinical protocol. MRAH, RRDL, JBG, AGMR collected all the samples. GVJS performed all the DNA extractions. GVJS, GMJF, PCVR, BM, LDVJ and LCC analyzed and interpreted all the data. GVJS, BM, LDVJ and LCC wrote the manuscript.

\section{Acknowledgements}

First, we wish to express our deepest gratitude to the families that accepted the invitation to participate in this study, and to the nurses and lactation consultants at the study hospital. We thank critical discussions with Dr. Marcos de Donato, Dra. Alejandra Chavez-Santoscoy and Dra. Karina Gutierrez. In addition, we are thankful to Luz Angela González Salazar for CIRCOS plotting. This work was performed partially through The National Council for Science and Technology (CONACYT) financial support (GVJS postgrad scholarship) and Tecnológico de Monterrey seed grant ITESM-0021C21064. 


\section{References}

1. Honda K, Littman DR. The microbiota in adaptive immune homeostasis and disease. Nature. 2016;535:75-84.

2. van den Elsen LWJ, Garssen J, Burcelin R, Verhasselt V. Shaping the gut microbiota by breastfeeding: the gateway to allergy prevention? Front Pediatr. 2019;7:47.

3. Bardanzellu F, Fanos V, Reali A. Omics" in human colostrum and mature milk: Looking to old data with new eyes. Nutrients. 2017;9.

4. Anderson G, Vaillancourt C, Maes M, Reiter RJ. Breastfeeding and the gut-brain axis: is there a role for melatonin? Biomol Concepts. 2017;8:185-195.

5. Houghteling PD, Walker WA. Why is initial bacterial colonization of the intestine important to infants' and children's health? J Pediatr Gastroenterol Nutr. 2015;60:294-307.

6. Walker WA, lyengar RS. Breast milk, microbiota, and intestinal immune homeostasis. Pediatr Res. 2015;77:220-228.

7. Boix-Amorós A, Collado MC, Mira A. Relationship between milk microbiota, bacterial load, macronutrients, and human cells during lactation. Front Microbiol. 2016;7:492.

8. Moossavi S, Sepehri S, Robertson B, Bode L, Goruk S, Field CJ, et al. Composition and variation of the human milk microbiota are influenced by maternal and early-life factors. Cell Host Microbe. 2019;25:324-335.e4.

9. Williams JE, Carrothers JM, Lackey KA, Beatty NF, York MA, Brooker SL, et al. Human milk microbial community structure is relatively stable and related to variations in macronutrient and micronutrient intakes in healthy lactating women. J Nutr. 2017;147:1739-1748.

10. Davé V, Street K, Francis S, Bradman A, Riley L, Eskenazi B, et al. Bacterial microbiome of breast milk and child saliva from low-income Mexican-American women and children. Pediatr Res. 2016;79:846-854.

11. Patel SH, Vaidya YH, Patel RJ, Pandit RJ, Joshi CG, Kunjadiya AP. Culture independent assessment of human milk microbial community in lactational mastitis. Sci Rep. 2017;7:7804.

12. Hermansson H, Kumar H, Collado MC, Salminen S, Isolauri E, Rautava S. Breast milk microbiota is shaped by mode of delivery and intrapartum antibiotic exposure. Front Nutr. 2019;6:4.

13. Gomez-Gallego C, Garcia-Mantrana I, Salminen S, Collado MC. The human milk microbiome and factors influencing its composition and activity. Semin Fetal Neonatal Med 2016;21:400-405.

14. Ruiz L, García-Carral C, Rodriguez JM. Unfolding the human milk microbiome landscape in the omics era. Front Microbiol. 2019;10:1378.

15. Coustan DR, Lowe LP, Metzger BE, Dyer AR, International Association of Diabetes and Pregnancy Study Groups. The hyperglycemia and adverse pregnancy outcome (HAPO) study: paving the way for new diagnostic criteria for gestational diabetes mellitus. Am J Obstet Gynecol. 2010;202:654.e16. 
16. Plows JF, Stanley JL, Baker PN, Reynolds CM, Vickers MH. The pathophysiology of gestational diabetes mellitus. Int J Mol Sci. 2018;19.

17. Kumar R. Prenatal factors and the development of asthma. Curr Opin Pediatr. 2008;20:682-687.

18. Peters JL, Suglia SF, Platts-Mills TAE, Hosen J, Gold DR, Wright RJ. Relationships among prenatal aeroallergen exposure and maternal and cord blood IgE: project ACCESS. J Allergy Clin Immunol. 2009;123:1041-1046.

19. Bassols J, Serino M, Carreras-Badosa G, Burcelin R, Blasco-Baque V, Lopez-Bermejo A, et al. Gestational diabetes is associated with changes in placental microbiota and microbiome. Pediatr Res. 2016;80:777-784.

20. Collado MC, Isolauri E, Laitinen K, Salminen S. Distinct composition of gut microbiota during pregnancy in overweight and normal-weight women. Am J Clin Nutr. 2008;88:894-899.

21. Cabrera-Rubio R, Collado MC, Laitinen K, Salminen S, Isolauri E, Mira A. The human milk microbiome changes over lactation is shaped by maternal weight and mode of delivery. Am J Clin Nutr. 2012;96:544-551.

22. Garcia-Mantrana I, Collado MC. Obesity and overweight: Impact on maternal and milk microbiome and their role for infant health and nutrition. Mol Nutr Food Res. 2016;60:1865-1875.

23. Peila C, Gazzolo D, Bertino E, Cresi F, Coscia A. Influence of diabetes during pregnancy on human milk composition. Nutrients. 2020;12.

24. Dainelli L, Prieto-Patron A, Silva-Zolezzi I, Sosa-Rubi SG, Espino Y Sosa S, Reyes-Muñoz E, et al. Screening and management of gestational diabetes in Mexico: results from a survey of multilocation, multi-health care institution practitioners. Diabetes Metab Syndr Obes. 2018;11:105116.

25. Corona-Cervantes K, García-González I, Villalobos-Flores LE, Hernández-Quiroz F, Piña-Escobedo A, Hoyo-Vadillo $\mathrm{C}$, et al. Human milk microbiota associated with early colonization of the neonatal gut in Mexican newborns. PeerJ. 2020;8:e9205.

26. Jost T, Lacroix C, Braegger CP, Rochat F, Chassard C. Vertical mother-neonate transfer of maternal gut bacteria via breastfeeding. Environ Microbiol. 2014;16:2891-2904.

27. Murphy K, Curley D, O'Callaghan TF, O'Shea C-A, Dempsey EM, O'Toole PW, et al. The composition of human milk and infant faecal microbiota over the first three months of life: A pilot study. Sci Rep. 2017;7:40597.

28. Kumar H, du Toit E, Kulkarni A, Aakko J, Linderborg KM, Zhang Y, et al. Distinct patterns in human milk microbiota and fatty acid profiles across specific geographic locations. Front Microbiol. 2016;7:1619.

29. Fernandes AD, Macklaim JM, Linn TG, Reid G, Gloor GB. ANOVA-like differential expression (ALDEx) analysis for mixed population RNA-Seq. PLoS One. 2013;8:e67019.

30. Chen P-W, Lin Y-L, Huang M-S. Profiles of commensal and opportunistic bacteria in human milk from healthy donors in Taiwan. J Food Drug Anal. 2018;26:1235-1244. 
31. Jost T, Lacroix C, Braegger C, Chassard C. Assessment of bacterial diversity in breast milk using culture-dependent and culture-independent approaches. Br J Nutr. 2013;110:1253-1262.

32. LeMay-Nedjelski L, Copeland J, Wang PW, Butcher J, Unger S, Stintzi A, et al. Methods and strategies to examine the human breastmilk microbiome. Methods Mol Biol. 2018;1849:63-86.

33. Douglas CA, Ivey KL, Papanicolas LE, Best KP, Muhlhausler BS, Rogers GB. DNA extraction approaches substantially influence the assessment of the human breast milk microbiome. Sci Rep. 2020;10:123.

34. de Goffau MC, Lager S, Salter SJ, Wagner J, Kronbichler A, Charnock-Jones DS, et al. Recognizing the reagent microbiome. Nat Microbiol. 2018;3:851-853.

35. Nogales B, Moore ER, Llobet-Brossa E, Rossello-Mora R, Amann R, Timmis KN. Combined use of $16 \mathrm{~S}$ ribosomal DNA and 16S rRNA to study the bacterial community of polychlorinated biphenyl-polluted soil. Appl Environ Microbiol. 2001;67:1874-1884.

36. Dewhirst FE, Klein EA, Thompson EC, Blanton JM, Chen T, Milella L, et al. The canine oral microbiome. PLoS One. 2012;7:e36067.

37. Camanocha A, Dewhirst FE. Host-associated bacterial taxa from Chlorobi, Chloroflexi, GN02, Synergistetes, SR1, TM7, and WPS-2 Phyla/candidate divisions. J Oral Microbiol. 2014;6.

38. Lazarevic V, Gaïa N, Emonet S, Girard M, Renzi G, Despres L, et al. Challenges in the cultureindependent analysis of oral and respiratory samples from intubated patients. Front Cell Infect Microbiol. 2014;4:65.

39. Marsh P, Lewis M, Rogers H, Williams D, Wilson M. Chapter 3. The resident oral microbiota. In: Oral microbiology. 2016. page 26-50.

40. Chen T, Yu W-H, Izard J, Baranova OV, Lakshmanan A, Dewhirst FE. The Human Oral Microbiome Database: a web accessible resource for investigating oral microbe taxonomic and genomic information. Database (Oxford). 2010;2010:baq013.

41. Prevaes SMPJ, de Winter-de Groot KM, Janssens HM, de Steenhuijsen Piters WAA, Tramper-Stranders $\mathrm{GA}$, Wyllie $\mathrm{AL}$, et al. Development of the nasopharyngeal microbiota in infants with cystic fibrosis. Am J Respir Crit Care Med. 2016;193:504-515.

42. Fernández L, Langa S, Martín V, Maldonado A, Jiménez E, Martín R, et al. The human milk microbiota: origin and potential roles in health and disease. Pharmacol Res. 2013;69:1-10.

43. Markle JGM, Frank DN, Mortin-Toth S, Robertson CE, Feazel LM, Rolle-Kampczyk U, et al. Sex differences in the gut microbiome drive hormone-dependent regulation of autoimmunity. Science. 2013;339:1084-1088.

44. Galante L, Milan AM, Reynolds CM, Cameron-Smith D, Vickers MH, Pundir S. Sex-specific human milk composition: the role of infant sex in determining early life nutrition. Nutrients. 2018;10.

45. Raju SC, Lagström S, Ellonen P, de Vos WM, Eriksson JG, Weiderpass E, et al. Gender-specific associations between saliva microbiota and body size. Front Microbiol. 2019;10:767. 
46. Koren O, Goodrich JK, Cullender TC, Spor A, Laitinen K, Bäckhed HK, et al. Host remodeling of the gut microbiome and metabolic changes during pregnancy. Cell. 2012;150:470-480.

47. Jost T, Lacroix $C$, Braegger $C$, Chassard $C$. Stability of the maternal gut microbiota during late pregnancy and early lactation. Curr Microbiol. 2014;68:419-427.

48. lebba V, Santangelo F, Totino V, Nicoletti M, Gagliardi A, De Biase RV, et al. Higher prevalence and abundance of Bdellovibrio bacteriovorus in the human gut of healthy subjects. PLoS One. 2013;8:e61608.

49. Mosca A, Leclerc M, Hugot JP. Gut microbiota diversity and human diseases: should we reintroduce key predators in our ecosystem? Front Microbiol. 2016;7:455.

50. Gagliardi A, Totino V, Cacciotti F, lebba V, Neroni B, Bonfiglio G, et al. Rebuilding the gut microbiota ecosystem. Int J Environ Res Public Health. 2018;15.

51. Eckburg PB, Bik EM, Bernstein CN, Purdom E, Dethlefsen L, Sargent M, et al. Diversity of the human intestinal microbial flora. Science. 2005;308:1635-1638.

52. Fredricks DN, Fiedler TL, Marrazzo JM. Molecular identification of bacteria associated with bacterial vaginosis. N Engl J Med. 2005;353:1899-1911.

53. Bor B, Bedree JK, Shi W, McLean JS, He X. Saccharibacteria (TM7) in the human oral microbiome. J Dent Res. 2019;98:500-509.

54. Lif Holgerson P, Harnevik L, Hernell O, Tanner ACR, Johansson I. Mode of birth delivery affects oral microbiota in infants. J Dent Res. 2011;90:1183-1188.

55. Brinig MM, Lepp PW, Ouverney CC, Armitage GC, Relman DA. Prevalence of bacteria of division TM7 in human subgingival plaque and their association with disease. Appl Environ Microbiol. 2003;69:1687-1694.

56. Kuehbacher T, Rehman A, Lepage P, Hellmig S, Fölsch UR, Schreiber S, et al. Intestinal TM7 bacterial phylogenies in active inflammatory bowel disease. J Med Microbiol. 2008;57:1569-1576.

57. Ferrocino I, Ponzo V, Gambino R, Zarovska A, Leone F, Monzeglio C, et al. Changes in the gut microbiota composition during pregnancy in patients with gestational diabetes mellitus (GDM). Sci Rep. 2018;8:12216.

58. Cortez RV, Taddei CR, Sparvoli LG, Ângelo AGS, Padilha M, Mattar R, et al. Microbiome and its relation to gestational diabetes. Endocrine. 2019;64:254-264.

59. Zheng W, Xu Q, Huang W, Yan Q, Chen Y, Zhang L, et al. Gestational diabetes mellitus is associated with reduced dynamics of gut microbiota during the first half of pregnancy. mSystems. 2020;5.

60. Asnicar F, Manara S, Zolfo M, Truong DT, Scholz M, Armanini F, et al. Studying vertical microbiome transmission from mothers to infants by strain-level metagenomic profiling. mSystems. 2017;2.

61. Wang J, Zheng J, Shi W, Du N, Xu X, Zhang Y, et al. Dysbiosis of maternal and neonatal microbiota associated with gestational diabetes mellitus. Gut. 2018;67:1614-1625.

62. Crusell MKW, Hansen TH, Nielsen T, Allin KH, Rühlemann MC, Damm P, et al. Gestational diabetes is associated with change in the gut microbiota composition in third trimester of pregnancy and 
postpartum. Microbiome. 2018;6:89.

63. Pujalte MJ, Lucena T, Ruvira MA, Arahal DR, Macián MC. The family Rhodobacteraceae. In: Rosenberg E, DeLong EF, Lory S, Stackebrandt E, Thompson F, editors. The Prokaryotes. Berlin, Heidelberg: Springer Berlin Heidelberg; 2014. page 439-512.

64. Hospodsky D, Pickering AJ, Julian TR, Miller D, Gorthala S, Boehm AB, et al. Hand bacterial communities vary across two different human populations. Microbiology (Reading, Engl.). 2014;160:1144-1152.

65. Wang Y, Liu Y, Bai J, Chen X. The effect of maternal postpartum practices on infant gut microbiota: A chinese cohort study. Microorganisms. 2019;7.

66. Sangster W, Hegarty JP, Schieffer KM, Wright JR, Hackman J, Toole DR, et al. Bacterial and fungal microbiota changes distinguish $C$. difficile infection from other forms of diarrhea: Results of a prospective inpatient study. Front Microbiol. 2016;7:789.

67. Douglas GM, Maffei VJ, Zaneveld JR, Yurgel SN, Brown JR, Taylor CM, et al. PICRUSt2 for prediction of metagenome functions. Nat Biotechnol. 2020;38:685-688.

68. Segata N, Izard J, Waldron L, Gevers D, Miropolsky L, Garrett WS, et al. Metagenomic biomarker discovery and explanation. Genome Biol. 2011;12:R60.

69. Caspi R, Billington R, Ferrer L, Foerster H, Fulcher CA, Keseler IM, et al. The MetaCyc database of metabolic pathways and enzymes and the BioCyc collection of pathway/genome databases. Nucleic Acids Res. 2016;44:D471-80.

70. Huynh J, Xiong G, Bentley-Lewis R. A systematic review of metabolite profiling in gestational diabetes mellitus. Diabetologia. 2014;57:2453-2464.

71. Much D, Beyerlein A, Kindt A, Krumsiek J, Stückler F, Rossbauer M, et al. Lactation is associated with altered metabolomic signatures in women with gestational diabetes. Diabetologia. 2016;59:21932202.

72. Zhao L, Wang M, Li J, Bi Y, Li M, Yang J. Association of circulating branched-chain amino acids with gestational diabetes mellitus: A meta-analysis. Int J Endocrinol Metab. 2019;17:e85413.

73. Much D, Beyerlein A, Roßbauer M, Hummel S, Ziegler A-G. Beneficial effects of breastfeeding in women with gestational diabetes mellitus. Mol Metab. 2014;3:284-292.

74. Koletzko B. Human milk lipids. Ann Nutr Metab. 2016;69 Suppl 2:28-40.

75. Costa SMR, Isganaitis E, Matthews TJ, Hughes K, Daher G, Dreyfuss JM, et al. Maternal obesity programs mitochondrial and lipid metabolism gene expression in infant umbilical vein endothelial cells. Int J Obes. 2016;40:1627-1634.

76. Panagos PG, Vishwanathan R, Penfield-Cyr A, Matthan NR, Shivappa N, Wirth MD, et al. Breastmilk from obese mothers has pro-inflammatory properties and decreased neuroprotective factors. J Perinatol. 2016;36:284-290.

77. Sorrow P, Maguire R, Murphy SK, Belcher SM, Hoyo C. Elevated metabolites of acetaminophen in cord blood of children with obesity. Pediatr Obes. 2019;14. 
78. Sobota-Grzeszyk A, Kuźmicki M, Szamatowicz J. Myoinositol in the prevention of gestational diabetes mellitus: is it sensible? J Diabetes Res. 2019;2019:3915253.

79. Formoso G, Baldassarre MPA, Ginestra F, Carlucci MA, Bucci I, Consoli A. Inositol and antioxidant supplementation: Safety and efficacy in pregnancy. Diabetes Metab Res Rev. 2019;35:e3154.

80. Croze ML, Soulage CO. Potential role and therapeutic interests of myo-inositol in metabolic diseases. Biochimie. 2013;95:1811-1827.

81. Santamaria A, Di Benedetto A, Petrella E, Pintaudi B, Corrado F, D’Anna R, et al. Myo-inositol may prevent gestational diabetes onset in overweight women: a randomized, controlled trial. J Matern Fetal Neonatal Med. 2016;29:3234-3237.

82. Tessler M, Neumann JS, Afshinnekoo E, Pineda M, Hersch R, Velho LFM, et al. Large-scale differences in microbial biodiversity discovery between $16 \mathrm{~S}$ amplicon and shotgun sequencing. Sci Rep. 2017;7:6589.

83. Jiménez E, de Andrés J, Manrique M, Pareja-Tobes P, Tobes R, Martínez-Blanch JF, et al. Metagenomic analysis of milk of healthy and mastitis-suffering women. J Hum Lact. 2015;31:406415.

84. Bolyen E, Rideout JR, Dillon MR, Bokulich NA, Abnet CC, Al-Ghalith GA, et al. Reproducible, interactive, scalable and extensible microbiome data science using QIIME 2. Nat Biotechnol. 2019;37:852-857.

85. Callahan BJ, McMurdie PJ, Rosen MJ, Han AW, Johnson AJA, Holmes SP. DADA2: High-resolution sample inference from Illumina amplicon data. Nat Methods. 2016;13:581-583.

86. Bokulich NA, Kaehler BD, Rideout JR, Dillon M, Bolyen E, Knight R, et al. Optimizing taxonomic classification of marker-gene amplicon sequences with QIIME 2's q2-feature-classifier plugin. Microbiome. 2018;6:90.

87. Pruesse E, Quast C, Knittel K, Fuchs BM, Ludwig W, Peplies J, et al. SILVA: a comprehensive online resource for quality checked and aligned ribosomal RNA sequence data compatible with ARB. Nucleic Acids Res. 2007;35:7188-7196.

88. Katoh K, Misawa K, Kuma K, Miyata T. MAFFT: a novel method for rapid multiple sequence alignment based on fast Fourier transform. Nucleic Acids Res. 2002;30:3059-3066.

89. Price MN, Dehal PS, Arkin AP. FastTree 2 - approximately maximum-likelihood trees for large alignments. PLoS One. 2010;5:e9490.

90. Chong J, Liu P, Zhou G, Xia J. Using MicrobiomeAnalyst for comprehensive statistical, functional, and meta-analysis of microbiome data. Nat Protoc. 2020;15:799-821.

\section{Supplementary Table}

A supplementary table was not provided with this version of the manuscript.

\section{Figures}


A)

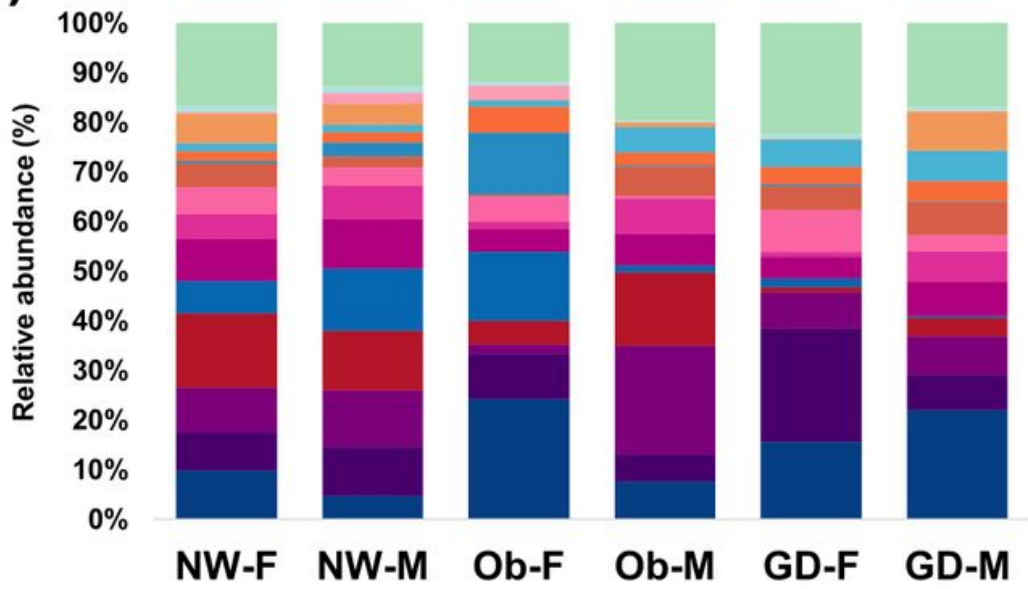

Proteobacteria

- Rhizobiaceae

Burkholderiaceae

Sphingomonadaceae

Xanthomonadaceae

Xanthobacteraceae

Neisseriaceae

\section{Firmicutes}

- Staphylococcaceae

Streptococcaceae

Bacillales: Family XI

Clostridiales: Family XI

Lactobacillales: P5D1-392

Bacillaceae

B)
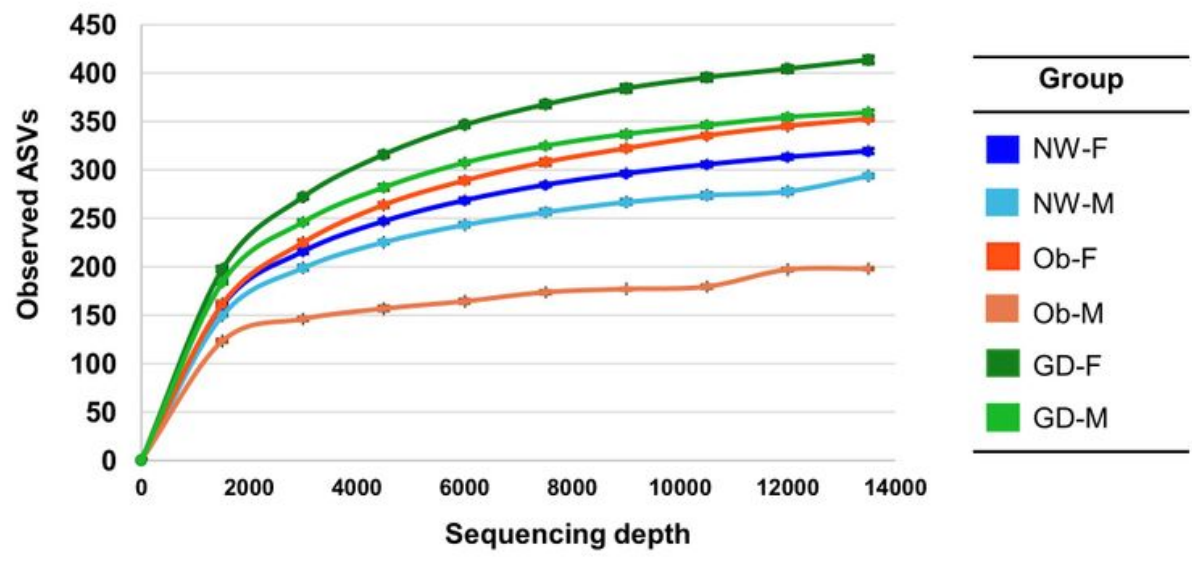

Figure 1

Bacterial diversity of breast milk samples. A) Taxonomic profile at family level divided by study subgroups (maternal health condition and gender of the newborn). B) Rarefaction curves from subgroups of breastmilk samples relating the sequencing depth and the estimated number of bacteria. ASVs, amplicon sequence variants; NW-F, healthy normal weight - female $(n=8)$; NW-M, healthy normal weight - male ( $n=10)$; Ob-F, obesity positive GDM negative- female $(n=8) ; O b-M$, obesity positive GDM negative - male ( $n=4)$; GD-F, GDM positive obesity negative - female baby ( $n=6)$; GD-M, GDM positive obesity negative - male $(n=7)$. 
A)

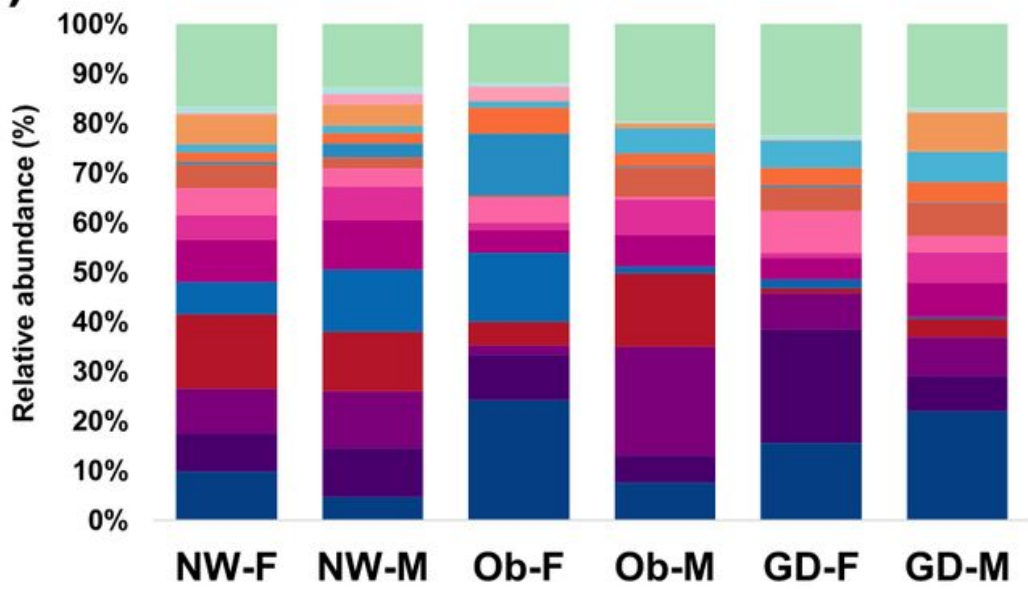

Proteobacteria

Rhizobiaceae

Burkholderiaceae

Sphingomonadaceae

Xanthomonadaceae

Xanthobacteraceae

Neisseriaceae

Firmicutes

- Staphylococcaceae

Streptococcaceae

Bacillales: Family XI

Clostridiales: Family XI

Lactobacillales: P5D1-392

Bacillaceae

B)
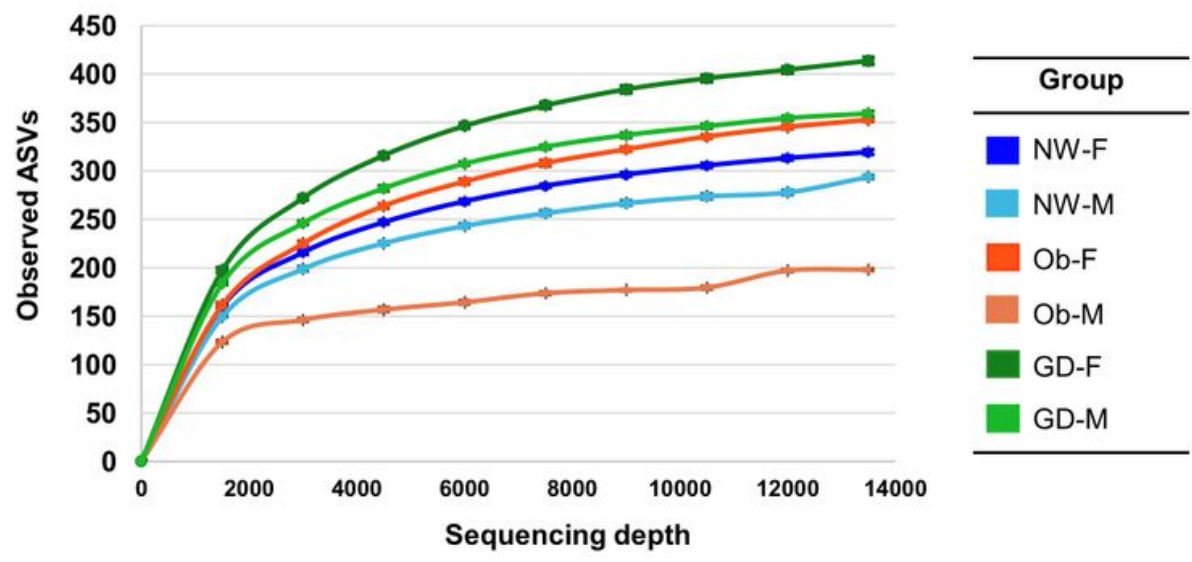

Figure 1

Bacterial diversity of breast milk samples. A) Taxonomic profile at family level divided by study subgroups (maternal health condition and gender of the newborn). B) Rarefaction curves from subgroups of breastmilk samples relating the sequencing depth and the estimated number of bacteria. ASVs, amplicon sequence variants; NW-F, healthy normal weight - female $(n=8)$; NW-M, healthy normal weight - male ( $n=10)$; Ob-F, obesity positive GDM negative- female $(n=8) ; O b-M$, obesity positive GDM negative - male $(n=4)$; GD-F, GDM positive obesity negative - female baby $(n=6)$; GD-M, GDM positive obesity negative - male $(n=7)$. 


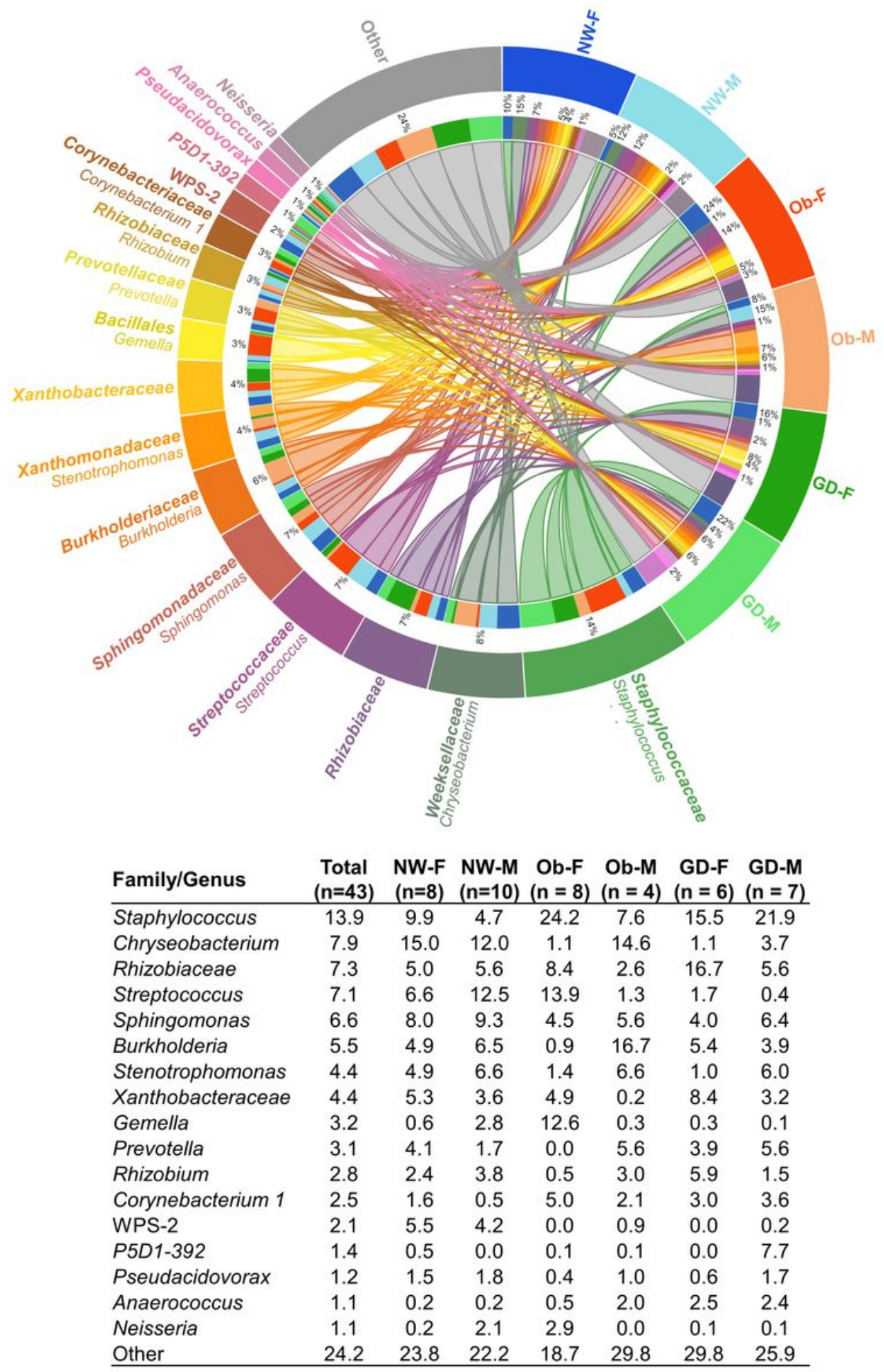

\section{Figure 2}

CIRCOS representation of the most abundant genera amongst healthy, obesity and Gestational Diabetes Mellitus groups. The table shows the relative abundance per subgroup of the 17 most abundant bacteria. "Other" represents all the taxa with less than $1 \%$ of total abundance. NW-F, healthy normal weight female; NW-M, healthy normal weight - male; Ob-F, obesity positive GDM negative- female; Ob-M, 
obesity positive GDM negative - male; GD-F, GDM positive obesity negative - female baby; GD-M, GDM positive obesity negative - male.

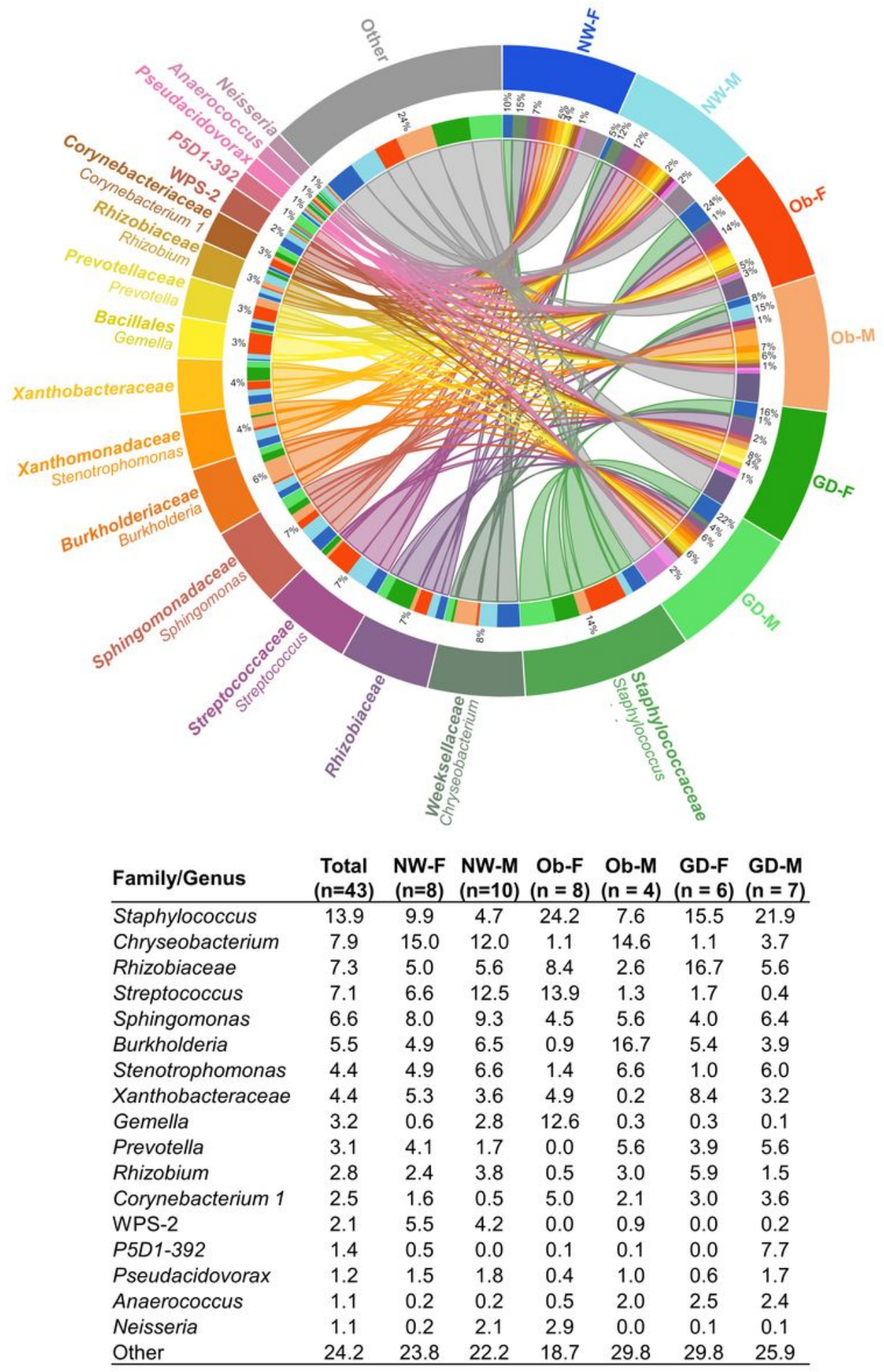

Figure 2

CIRCOS representation of the most abundant genera amongst healthy, obesity and Gestational Diabetes Mellitus groups. The table shows the relative abundance per subgroup of the 17 most abundant bacteria. "Other" represents all the taxa with less than $1 \%$ of total abundance. NW-F, healthy normal weight - 
female; NW-M, healthy normal weight - male; Ob-F, obesity positive GDM negative- female; Ob-M, obesity positive GDM negative - male; GD-F, GDM positive obesity negative - female baby; GD-M, GDM positive obesity negative - male.

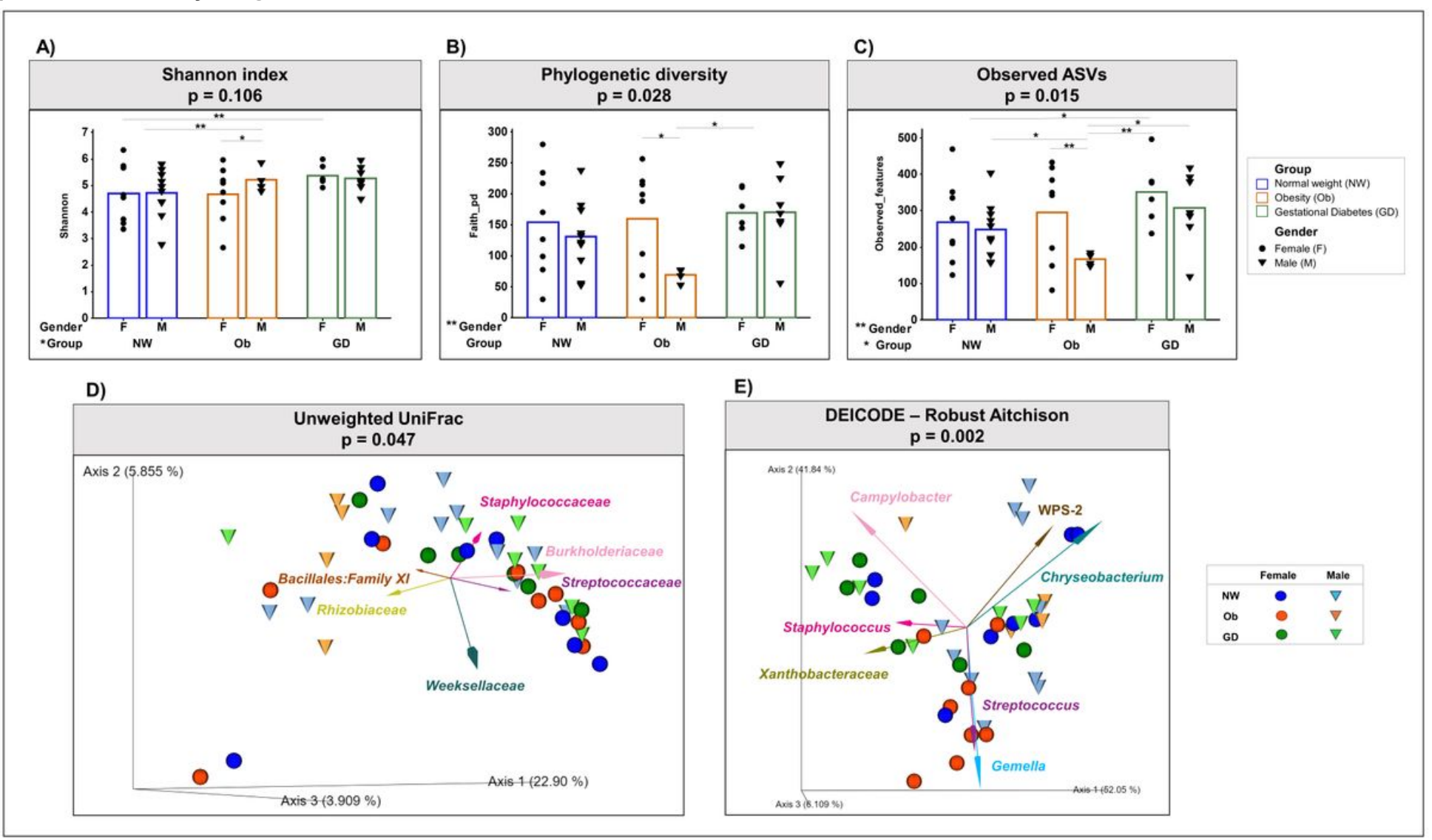

Figure 3

Alpha and Beta diversity indexes of breastmilk. A-C, Alpha diversity. D-E, Beta diversity. A) Shannon index. B) Number of observed ASVs. C) Phylogenetic diversity. All the Alpha indexes showed significant differences after a general linear model $(\mathrm{glm})$ with a confidence level of $95 \%(p \leq 0.05)$. Fisher test was implemented for comparisons. D) Unweighted principal coordinate analysis (PCoA) biplot of UniFrac distances with vectors at family level. E) Robust principal component analysis (RPCA) biplot using DEICODE (robust Aitchison). Beta indexes showed significant differences after being assessed by permutational ANOVA (999 permutations). ${ }^{\star} p \leq 0.10 ;{ }^{* \star} p \leq 0.05$. 


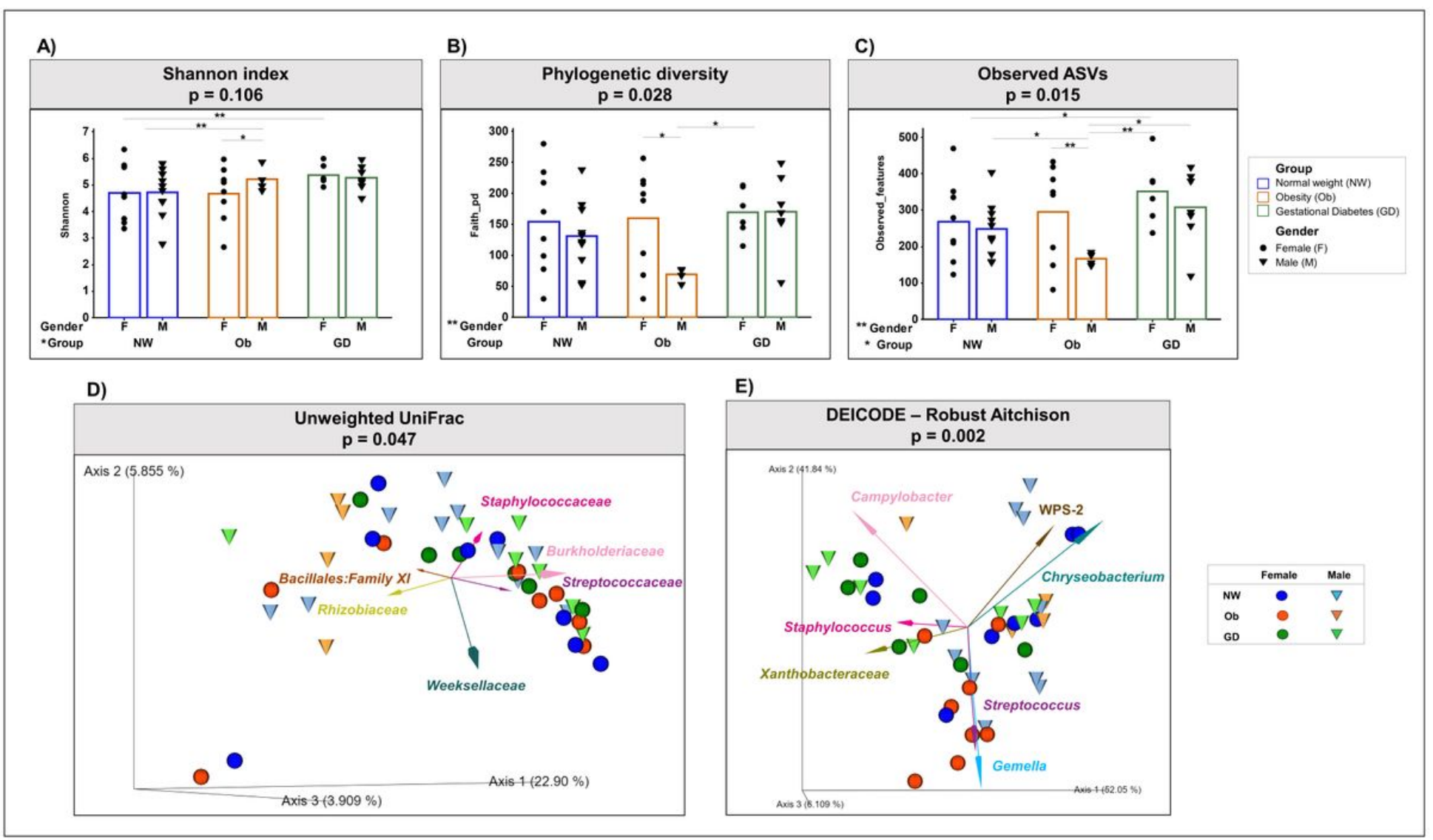

Figure 3

Alpha and Beta diversity indexes of breastmilk. A-C, Alpha diversity. D-E, Beta diversity. A) Shannon index. B) Number of observed ASVs. C) Phylogenetic diversity. All the Alpha indexes showed significant differences after a general linear model $(\mathrm{glm})$ with a confidence level of $95 \%(p \leq 0.05)$. Fisher test was implemented for comparisons. D) Unweighted principal coordinate analysis (PCoA) biplot of UniFrac distances with vectors at family level. E) Robust principal component analysis (RPCA) biplot using DEICODE (robust Aitchison). Beta indexes showed significant differences after being assessed by permutational ANOVA (999 permutations). ${ }^{*} \mathrm{p} \leq 0.10$; ${ }^{*} \mathrm{p} \leq 0.05$. 


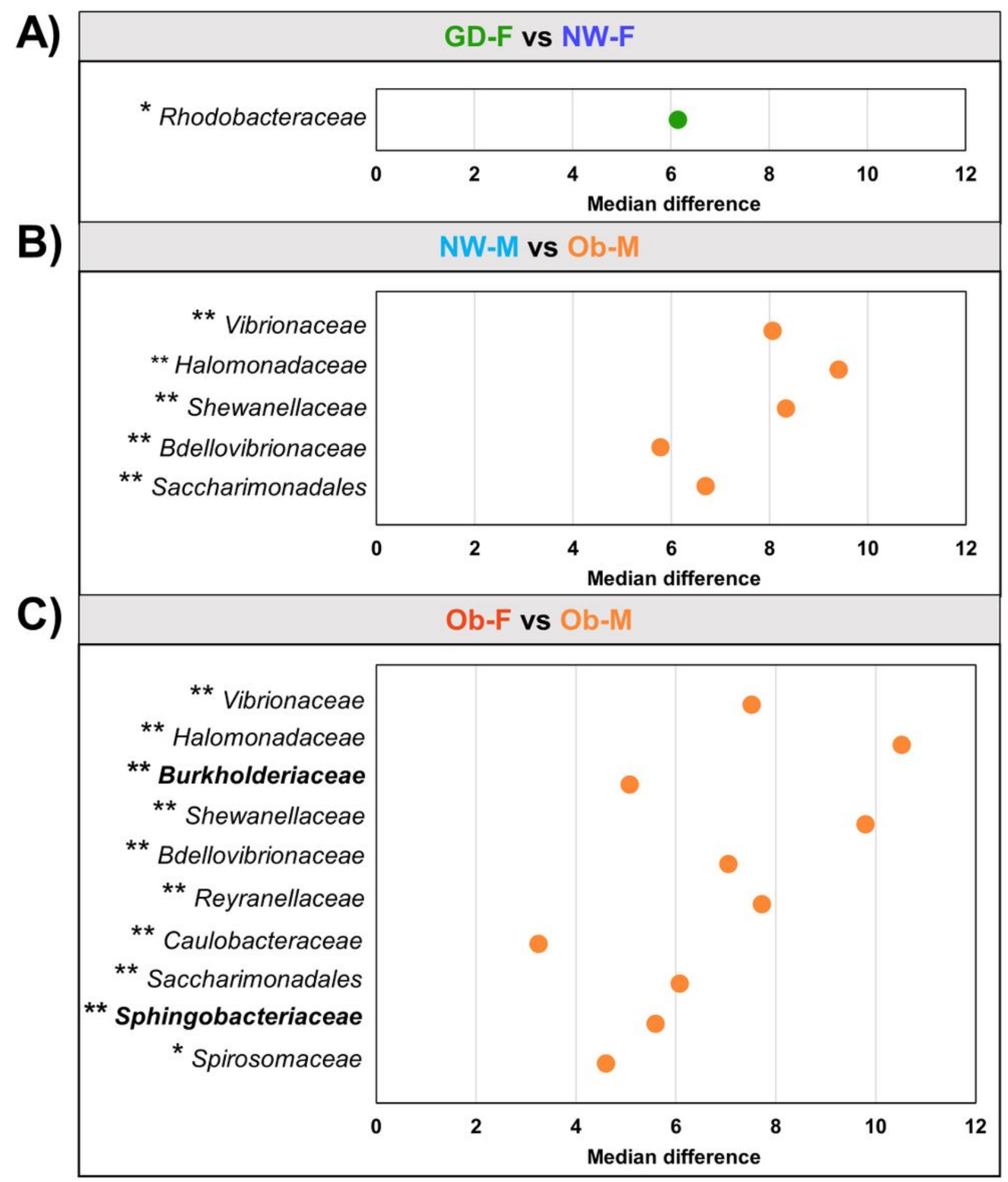

Figure 4

Differential bacteria at family level between subgroups. Each panel shows a plot illustrating differentially abundant taxon for each comparison and their median difference of centered log-ratio (clr) transformation, which indicates the dimension of the difference in abundance. Taxa with bold letters represent members of the core microbiota. Dots in the plot represent the median difference of significant features after a Welch's t test (adjusted p-value $\leq 0.1$ ) and effect size $\geq 1$. Dots are colored according to 
the subgroup that contained a greater fraction. A) Comparison of GDM-female versus healthy-female. B) Comparison of GDM-male versus obesity-male. C) Comparison of healthy-male versus obesity-male. D) Comparison of obesity-female versus obese-male. ${ }^{*} \mathrm{q} \leq 0.1 ;{ }^{* *} \mathrm{q} \leq 0.05$.

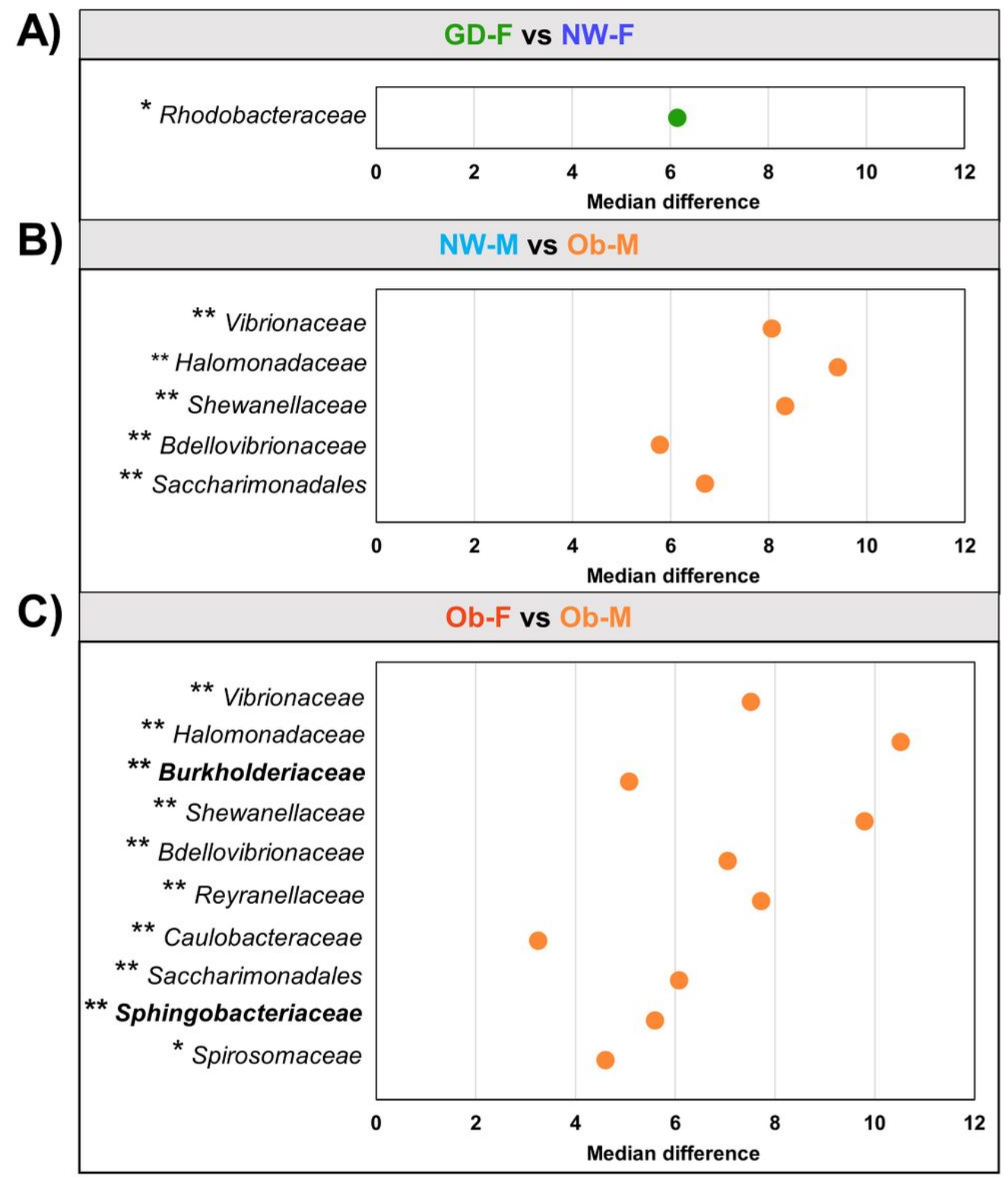

Figure 4

Differential bacteria at family level between subgroups. Each panel shows a plot illustrating differentially abundant taxon for each comparison and their median difference of centered log-ratio (clr) 
transformation, which indicates the dimension of the difference in abundance. Taxa with bold letters represent members of the core microbiota. Dots in the plot represent the median difference of significant features after a Welch's t test (adjusted $p$-value $\leq 0.1$ ) and effect size $\geq 1$. Dots are colored according to the subgroup that contained a greater fraction. A) Comparison of GDM-female versus healthy-female. B) Comparison of GDM-male versus obesity-male. C) Comparison of healthy-male versus obesity-male. D) Comparison of obesity-female versus obese-male. ${ }^{*} \mathrm{q} \leq 0.1 ;{ }^{* \star} \mathrm{q} \leq 0.05$.

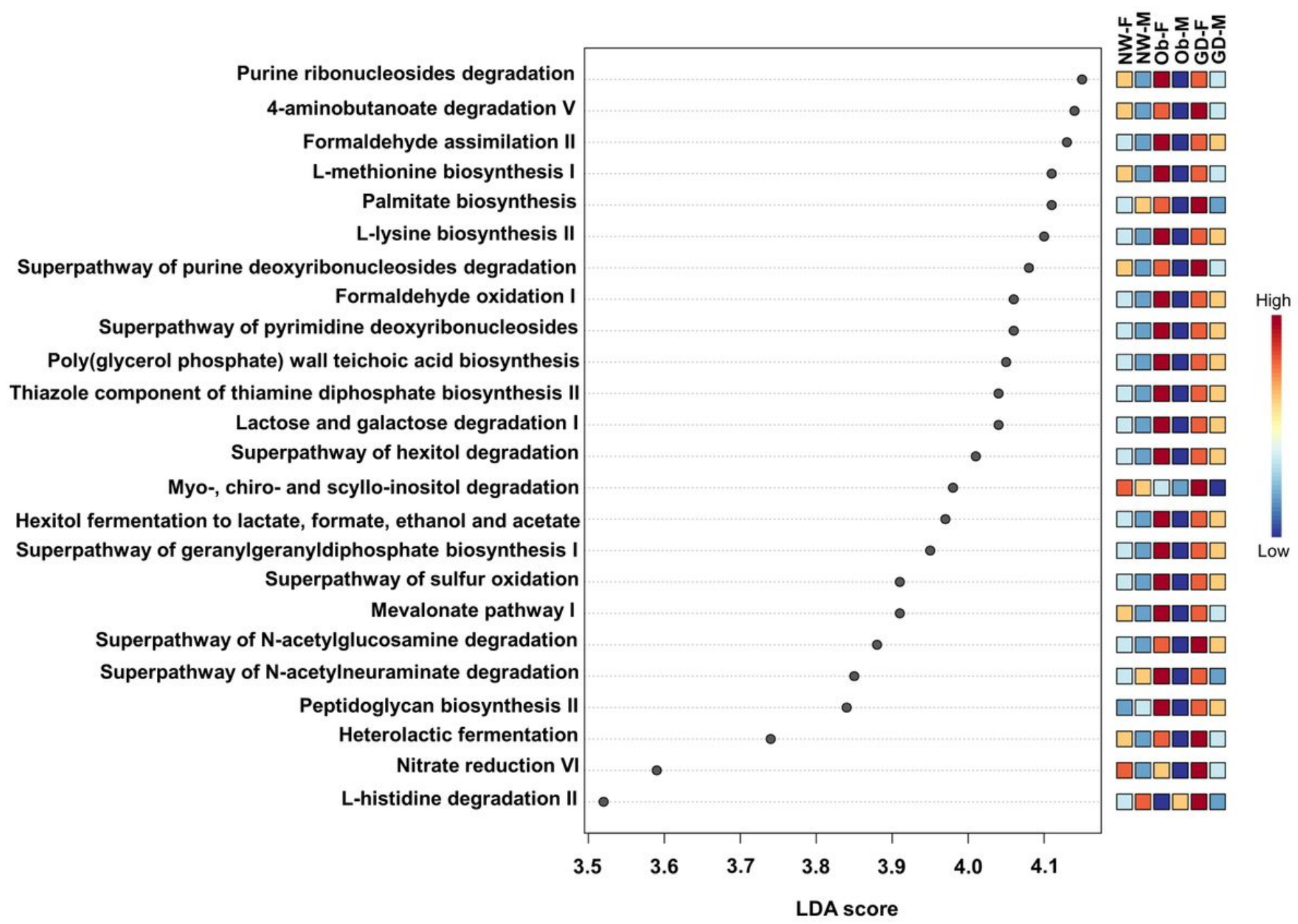

\section{Figure 5}

LEfSe analysis of the breastmilk's functional profiling prediction between subgroups. A significance pvalue $\leq 0.05$ and an effect size threshold of 3.5 were used for all Metacyc pathways evaluated. NW-F, healthy normal weight - female; NW-M, healthy normal weight - male; Ob-F, obesity positive GDM negative - female; Ob-M, obesity positive GDM negative - male; GD-F, GDM positive obesity negative female baby; GD-M, GDM positive obesity negative - male. 


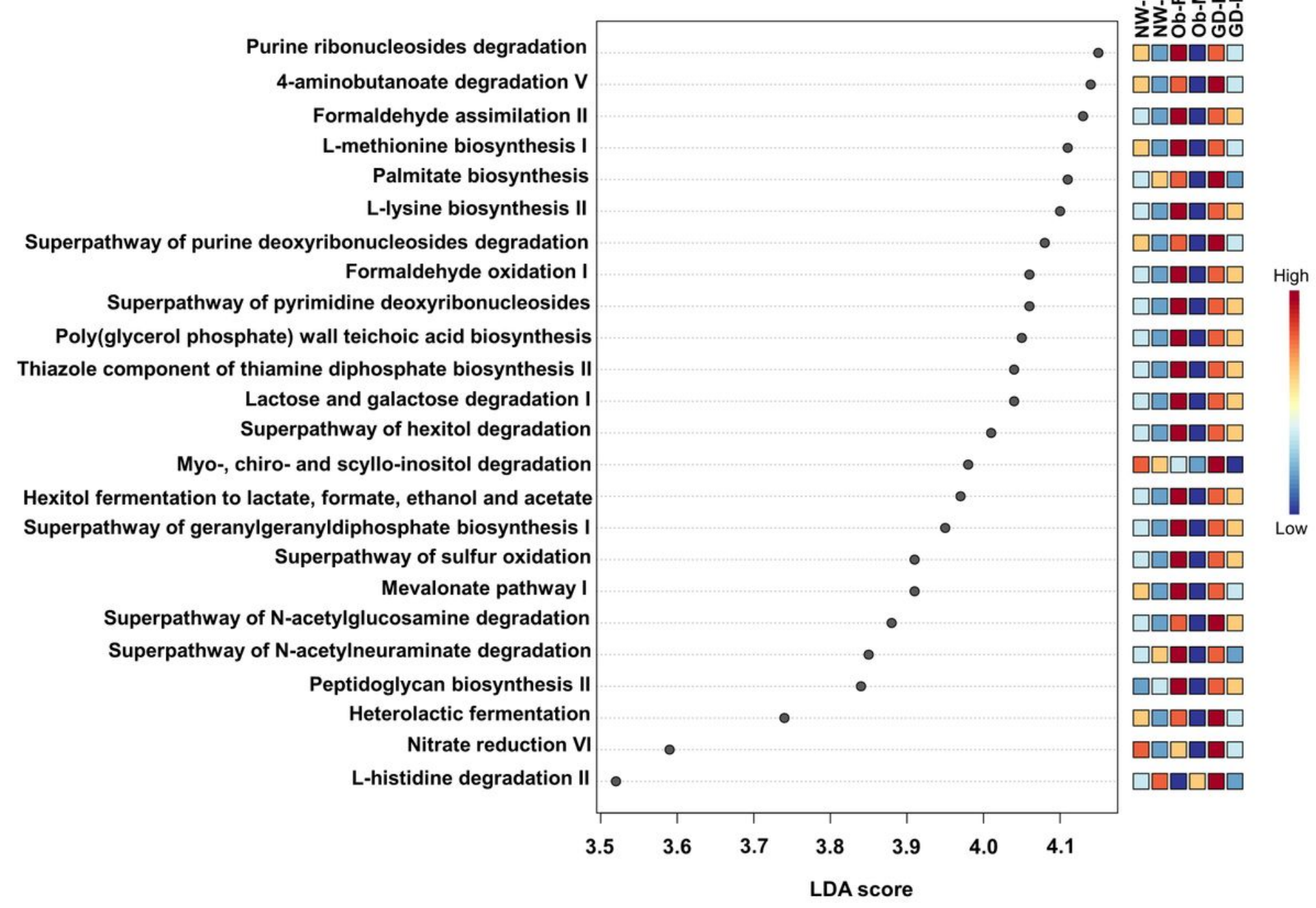

\section{Figure 5}

LEfSe analysis of the breastmilk's functional profiling prediction between subgroups. A significance pvalue $\leq 0.05$ and an effect size threshold of 3.5 were used for all Metacyc pathways evaluated. NW-F, healthy normal weight - female; NW-M, healthy normal weight - male; Ob-F, obesity positive GDM negative- female; Ob-M, obesity positive GDM negative - male; GD-F, GDM positive obesity negative female baby; GD-M, GDM positive obesity negative - male.

\section{Supplementary Files}

This is a list of supplementary files associated with this preprint. Click to download.

- SupplementaryFigure1.jpg

- SupplementaryFigure1.jpg 